\title{
Hom-Jordan and Hom-alternative bimodules
}

\author{
S. Attan, H. Hounnon, B. Kpamegan \\ Département de Mathématiques, Université d'Abomey-Calavi \\ 01 BP 4521, Cotonou 01, Bénin \\ syltane2010@yahoo.fr, hi.hounnon@fast.uac.bj, kpamegan_bernadin@yahoo.fr
}

Received March 21, 2019 and, in revised form, April 8, 2020

Presented by Consuelo Martínez Accepted April 15, 2020

\begin{abstract}
In this paper, Hom-Jordan and Hom-alternative bimodules are introduced. It is shown that Jordan and alternative bimodules are twisted via endomorphisms into Hom-Jordan and Homalternative bimodules respectively. Some relations between Hom-associative bimodules, Hom-Jordan and Hom-alternative bimodules are given.

Key words: Bimodules, alternative algebras, Jordan algebras, Hom-alternative algebras, HomJordan algebras, Hom-associative algebras.

AMS Subject Class. (2010): 17A30, 17B10, 17C50, 17D05.
\end{abstract}

\section{INTRODUCTION}

Algebras where the identities defining the structure are twisted by a homomorphism are called Hom-algebras. They have been intensively investigated in the literature recently. Hom-algebra started from Hom-Lie algebras introduced and discussed in [6, 10, 11, 12, motivated by quasi-deformations of Lie algebras of vector fields, in particular q-deformations of Witt and Virasoro algebras. Hom-associative algebras were introduced in [15] while Homalternative and Hom-Jordan algebras are introduced in [14], [23] as twisted generalizations of alternative and Jordan algebra respectively. The reader is referred to [20] for applications of alternative algebras to projective geometry, buildings, and algebraic groups and to [4, 9, 16, 19] for discussions about the important roles of Jordan algebras in physics, especially quantum mechanics.

The anti-commutator of a Hom-alternative algebra gives rise to a HomJordan algebra [23]. Starting with a Hom-alternative algebra $(A, \cdot, \alpha)$, it is known that the Jordan product

$$
x * y=\frac{1}{2}(x \cdot y+y \cdot x)
$$

gives a Hom-Jordan algebra $A^{+}=(A, *, \alpha)$. In other words, Hom-alternative algebras are Hom-Jordan-admissible [23]. 
The notion of bimodule for a class of algebras defined by multilinear identities has been introduced by Eilenberg [3. If $\mathcal{H}$ is in the class of associative algebras or in the one of Lie algebras then this notion is the familiar one for which we are in possession of well-worked theories. The study of bimodule (or representation) of Jordan algebras was initiated by N. Jacobson [7]. Subsequently the alternative case was considered by Schafer [17].

Modules over an ordinary algebra has been extended to the ones of Homalgebras in many works [2, 18, 21, 22].

The aim of this paper is to introduce Hom-alternative bimodules and HomJordan bimodules and to discuss about some findings. The paper is organized as follows. In section two, we recall basic notions related to Hom-algebras and modules over Hom-associative algebras. Section three is devoted to the introduction of Hom-alternative bimodules . Proposition 3.7 shows that from a given Hom-alternative bimodule, a sequence of this kind of bimodules can be obtained. Theorem 3.8 establishes that, an alternative bimodule gives rise to a bimodule over the corresponding twisted algebra. It is also proved that a direct sum of a Hom-alternative algebra and a module over this Homalgebra is again a Hom-alternative algebra (Theorem 3.11). In section four, we introduce Hom-Jordan modules and attest similar results as in the previous section. Furthermore, it is proved that a Hom-Jordan special left and right module, with an additional condition, has a bimodule structure over this Homalgebra (Theorem 4.10). Finally, Proposition 4.12 shows that a bimodule over a Hom-associative algebra has a bimodule structure over its plus Homalgebra. All vector spaces are assumed to be over a fixed ground field $\mathbb{K}$ of characteristic 0 .

\section{Preliminaries}

We recall some basic notions introduced in 6, 15, 21, related to Homalgebras and while dealing of any binary operation we will use juxtaposition in order to reduce the number of braces, i.e., e.g., for ".", $x y \cdot \alpha(z)$ means $(x \cdot y) \cdot \alpha(z)$. Also, for the map $\mu: A^{\otimes 2} \rightarrow A$, we will write sometimes $\mu(a \otimes b)$ as $\mu(a, b)$ or $a b$ for $a, b \in A$ and if $V$ is another vector space, $\tau_{1}: A \otimes V \rightarrow V \otimes A$ (resp. $\tau_{2}: V \otimes A \rightarrow A \otimes V$ ) denote the twist isomorphism $\tau_{1}(a \otimes v)=v \otimes a$ (resp. $\left.\tau_{2}(v \otimes a)=a \otimes v\right)$.

Definition 2.1. A Hom-module is a pair $\left(M, \alpha_{M}\right)$ consisting of a $\mathbb{K}$ module $M$ and a linear self-map $\alpha_{M}: M \rightarrow M$. A morphism $f:\left(M, \alpha_{M}\right) \rightarrow$ $\left(N, \alpha_{N}\right)$ of Hom-modules is a linear map $f: M \rightarrow N$ such that $f \circ \alpha_{M}=\alpha_{N} \circ f$. 
Definition 2.2. ([15, 21]) A Hom-algebra is a triple $\left(A, \mu_{A}, \alpha_{A}\right)$ in which $\left(A, \alpha_{A}\right)$ is a Hom-module, $\mu: A^{\otimes 2} \rightarrow A$ is a linear map. The Homalgebra $(A, \mu, \alpha)$ is said to be multiplicative if $\alpha \circ \mu=\mu \circ \alpha^{\otimes 2}$ (multiplicativity). A morphism $f:\left(A, \mu_{A}, \alpha_{A}\right) \rightarrow\left(B, \mu_{B}, \alpha_{B}\right)$ of Hom-algebras is a morphism of the underlying Hom-modules such that $f \circ \mu_{A}=\mu_{B} \circ f^{\otimes 2}$.

An important class of Hom-algebras that is considered here is the one of Hom-alternative algebras. These algebras have been introduced in [14] and more studied in [23].

Definition 2.3. Let $(A, \mu, \alpha)$ be a Hom-algebra.

(i) The Hom-associator of $A$ is the linear map $a s_{A}: A^{\otimes 3} \rightarrow A$ defined as $a s_{A}=\mu \circ(\mu \otimes \alpha-\alpha \otimes \mu)$. A multiplicative Hom-algebra $(A, \mu, \alpha)$ is said to be Hom-associative algebra if $a s_{A}=0$.

(ii) A Hom-alternative algebra [14 is a multiplicative Hom-algebra $(A, \mu, \alpha)$ that satisfies

$$
\begin{aligned}
& a s_{A}(x, x, y)=0 \quad \text { (left Hom-alternativity), } \\
& a s_{A}(x, y, y)=0 \quad \text { (right Hom-alternativity) }
\end{aligned}
$$

for all $x, y \in A$.

(iii) Let $(A, \mu, \alpha)$ be a Hom-alternative algebra. A Hom-subalgebra of $(A, \mu, \alpha)$ is a linear subspace $H$ of $A$, which is closed for the multiplication $\mu$ and invariant by $\alpha$, that is, $\mu(x, y) \in H$ and $\alpha(x) \in H$ for all $x, y \in H$. If furthermore $\mu(a, b) \in H$ and $\mu(b, a) \in H$ for all $(a, b) \in A \times H$, then $H$ is called a two-sided Hom-ideal of $A$.

Now, we prove:

Proposition 2.4. Let $(A, \mu, \alpha)$ be a Hom-alternative algebra and $I$ be a two-sided Hom-ideal of $(A, \mu, \alpha)$. Then $(A / I, \bar{\mu}, \bar{\alpha})$ is a Hom-alternative algebra where $\bar{\mu}(\bar{x}, \bar{y})=\overline{\mu(x, y)}$ and $\bar{\alpha}(\bar{x})=\alpha(x)$ for all $\bar{x}, \bar{y} \in A / I$.

Proof. First, note that the multiplicativity of $\bar{\mu}$ with respect to $\bar{\alpha}$ follows from the one of $\mu$ with respect to $\alpha$. Next, pick $\bar{x}, \bar{y} \in A / I$. Then the left Hom-alternativity (1) in $(A / I, \bar{\mu}, \bar{\alpha})$ is proved as follows

$$
\begin{aligned}
a s_{A / I}(\bar{x}, \bar{x}, \bar{y}) & =\bar{\mu}(\bar{\mu}(\bar{x}, \bar{x}), \bar{\alpha}(\bar{y}))-\bar{\mu}(\bar{\alpha}(\bar{x}), \bar{\mu}(\bar{x}, \bar{y}) \\
& =\overline{\mu(\mu(x, x) \alpha(y))-\mu(\alpha(x), \mu(x, y))}=\overline{\left.a s_{A}(x, x, y)\right)}=\overline{0} .
\end{aligned}
$$

Similarly, we get (2) and therefore $(A / I, \bar{\mu}, \bar{\alpha})$ is a Hom-alternative algebra. 
As Hom-alternative algebras, Hom-Jordan algebras are fundamental objects of this paper. They appear as cousins of Hom-alternative algebras and these two Hom-algebras are related as Jordan and alternative algebras.

Definition 2.5. ([23]) (i) A Hom-Jordan algebra is a multiplicative Hom-algebra $(A, \mu, \alpha)$ such that $\mu \circ \tau=\mu$ (commutativity of $\mu$ ) and the so-called Hom-Jordan identity holds

$$
a s_{A}(\mu(x, x,), \alpha(y), \alpha(x))=0, \forall(x, y) \in A^{2}
$$

where, $\tau: A^{\otimes 2} \rightarrow A^{\otimes 2}, \tau(a \otimes b)=b \otimes a$, is the twist isomorphism.

(ii) Let $(A, \mu, \alpha)$ be a Hom-Jordan algebra. A Hom-subalgebra of $(A, \mu, \alpha)$ is a linear subspace $H$ of $A$, which is closed for the multiplication $\mu$ and invariant by $\alpha$, that is, $\mu(x, y) \in H$ and $\alpha(x) \in H$ for all $x, y \in H$. If furthermore $\mu(a, b) \in H$ for all $(a, b) \in A \times H$, then $H$ is called a two-sided Hom-ideal (or simply Hom-ideal) of $A[5]$.

Similarly as a Hom-alternative algebra case, if $H$ is a Hom-ideal of a HomJordan algebra $(A, \mu, \alpha)$, then $(A / H, \bar{\mu}, \bar{\alpha})$ is a Hom-Jordan algebra where $\bar{\mu}(\bar{x}, \bar{y})=\overline{\mu(x, y)}$ for all $\bar{x}, \bar{y} \in A / H$ and $\bar{\alpha}: A / H \rightarrow A / H$ is naturally induced by $\alpha$, inherits a Hom-Jordan algebra structure, which is named quotient HomJordan algebra.

Remark 2.6. In [14 Makhlouf defined a Hom-Jordan algebra as a commutative multiplicative Hom-algebra satisfying $a s_{A}\left(x^{2}, y, \alpha(x)\right)=0$, which becomes the identity (3) if $y$ is replaced by $\alpha(y)$.

The proof of the following result can be found in [23] where the product $*$, differs from the one given here by a factor of $\frac{1}{2}$.

Proposition 2.7. Let $(A, \mu, \alpha)$ be a Hom-alternative algebra. Then $A^{+}$ $=(A, *, \alpha)$ is a Hom-Jordan algebra where $x * y=x y+y x$ for all $x, y \in A$.

ExAmple 2.8. From the eight-dimensional Hom-alternative algebra $O_{\alpha}=$ $\left(O, \mu_{\alpha}, \alpha\right)$ with basis $\left\{e_{0}, e_{1}, 2, e_{3}, e_{4}, e_{5}, e_{6}, e_{7}\right\}$ [23, Example 3.19], constructed from the octonion algebra which is an eight-dimensional alternative algebra, we obtain, the Hom-Jordan algebra $O_{\alpha}^{+}=\left(O, *=\mu_{\alpha}+\mu_{\alpha} \circ \tau, \alpha\right)$ where the non zero products are: $e_{0} * e_{0}=2 e_{0}, e_{0} * e_{1}=e_{1} * e_{0}=2 e_{5}, e_{0} * e_{2}=e_{2} * e_{0}=2 e_{6}$, $e_{0} * e_{3}=e_{3} * e_{0}=2 e_{7}, e_{0} * e_{4}=e_{4} * e_{0}=2 e_{1}, e_{0} * e_{5}=e_{5} * e_{0}=2 e_{2}$, $e_{0} * e_{6}=e_{6} * e_{0}=2 e_{3}, e_{0} * e_{7}=e_{7} * e_{0}=2 e_{4}, e_{1} * e_{1}=e_{2} * e_{2}=e_{3} * e_{3}=$ 
$e_{4} * e_{4}=e_{5} * e_{5}=e_{6} * e_{6}=e_{7} * e_{7}=-2 e_{0}$ and the twisting map $\alpha$ is given by $\alpha\left(e_{0}\right)=e_{0}, \alpha\left(e_{1}\right)=e_{5}, \alpha\left(e_{2}\right)=e_{6}, \alpha\left(e_{3}\right)=e_{7}, \alpha\left(e_{4}\right)=e_{1}, \alpha\left(e_{5}\right)=e_{2}$, $\alpha\left(e_{6}\right)=e_{3}, \alpha\left(e_{7}\right)=e_{4}$.

A. Makhlouf proved that the plus algebra of any Hom-associative algebra is a Hom-Jordan algebra as defined in [14]. Here, we prove the same result for the Hom-Jordan algebra as defined in [23] (see also Definition 2.5 above).

Proposition 2.9. Let $(A, \cdot, \alpha)$ be a Hom-associative algebra. Then $A^{+}=$ $(A, *, \alpha)$ is a Hom-Jordan algebra where $x * y=x y+y x$ for all $x, y \in A$.

Proof. The commutativity of $*$ is obvious. We compute the Hom-Jordan identity as follows:

$$
\begin{aligned}
a s_{A^{+}} & \left.x^{2}, \alpha(x), \alpha(y)\right) \\
= & \left(x^{2} * \alpha(y)\right) * \alpha^{2}(x)-\alpha\left(x^{2}\right) *(\alpha(y) * \alpha(x)) \\
= & \left(x^{2} \cdot \alpha(y)\right) \cdot \alpha^{2}(x)+\left(\alpha(y) \cdot x^{2}\right) \cdot \alpha^{2}(x)+\alpha^{2}(x) \cdot\left(x^{2} \cdot \alpha(y)\right) \\
& +\alpha^{2}(x) \cdot\left(\alpha(y) \cdot x^{2}\right)-\alpha\left(x^{2}\right) \cdot(\alpha(y) \cdot \alpha(x))-\alpha\left(x^{2}\right) \cdot(\alpha(x) \cdot \alpha(y)) \\
& -(\alpha(y) \cdot \alpha(x)) \cdot \alpha\left(x^{2}\right)-(\alpha(x) \cdot \alpha(y)) \cdot \alpha\left(x^{2}\right) \text { (by a direct computation) } \\
= & \left(\alpha(y) \cdot x^{2}\right) \cdot \alpha^{2}(x)+\alpha^{2}(x) \cdot\left(x^{2} \cdot \alpha(y)\right)-\alpha\left(x^{2}\right) \cdot(\alpha(x) \cdot \alpha(y)) \\
& -(\alpha(y) \cdot \alpha(x)) \cdot \alpha\left(x^{2}\right) \quad(\text { by the Hom-associativity) } \\
= & \left(\alpha(y) \cdot x^{2}\right) \cdot \alpha^{2}(x)+\alpha^{2}(x) \cdot\left(x^{2} \cdot \alpha(y)\right)-(\alpha(x) \cdot \alpha(x)) \cdot \alpha(x \cdot y) \\
& -\alpha(y x) \cdot(\alpha(x) \cdot \alpha(x)) \quad(\text { by the multiplicativity) } \\
= & \left(\alpha(y) \cdot x^{2}\right) \cdot \alpha^{2}(x)+\alpha^{2}(x) \cdot\left(x^{2} \cdot \alpha(y)\right)-\alpha^{2}(x) \cdot(\alpha(x) \cdot(x \cdot y)) \\
& -((y x) \cdot \alpha(x)) \cdot \alpha^{2}(x) \quad \text { (by the Hom-associativity) } \\
= & 0 \quad(\text { by the Hom-associativity). }
\end{aligned}
$$

Then $A^{+}=(A, *, \alpha)$ is a Hom-Jordan algebra.

Examples 2.10. (i) Consider the three-dimensional Hom-associative algebra $\mathcal{A}=\left(A, \mu_{A}, \alpha_{A}\right)$ over $\mathbb{K}$ with basis $\left(e_{1}, e_{2}, e_{3}\right)$ defined by $\mu_{A}\left(e_{1}, e_{1}\right)=e_{1}$, $\mu_{A}\left(e_{2}, e_{2}\right)=e_{2}, \mu_{A}\left(e_{3}, e_{3}\right)=e_{1}, \mu_{A}\left(e_{1}, e_{3}\right)=\mu_{A}\left(e_{3}, e_{1}\right)=-e_{3}$ and $\alpha_{A}\left(e_{1}\right)=$ $e_{1}, \alpha_{A}\left(e_{3}\right)=-e_{3}$ (see [24, Theorem 3.12], Hom-algebra $\left.A_{3}^{\prime 3}\right)$. Using the product $*$ in Proposition 2.9, the triple $\mathcal{A}^{+}=\left(A, *, \alpha_{A}\right)$ is Hom-Jordan algebra where, $e_{1} * e_{1}=2 e_{1}, e_{2} * e_{2}=2 e_{2}, e_{3} * e_{3}=2 e_{1}, e_{1} * e_{3}=e_{3} * e_{1}=-2 e_{3}$. 
(ii) From the three-dimensional Hom-associative algebra $\mathcal{B}=\left(B, \mu_{B}, \alpha_{B}\right)$ over $\mathbb{K}$ with basis $\left(e_{1}, e_{2}, e_{3}\right)$ defined by $\mu_{B}\left(e_{1}, e_{1}\right)=e_{1}, \mu_{B}\left(e_{2}, e_{2}\right)=e_{1}$, $\mu_{B}\left(e_{3}, e_{3}\right)=e_{3}, \mu_{B}\left(e_{1}, e_{2}\right)=\mu_{B}\left(e_{2}, e_{1}\right)=-e_{2}$ and $\alpha_{B}\left(e_{1}\right)=e_{1}, \alpha_{B}\left(e_{2}\right)=$ $-e_{2}$ (see [24, Theorem 3.12], Hom-algebra $\left.A_{5}^{\prime 3}\right)$. Then the triple $\mathcal{B}^{+}=(B, *$, $\alpha_{B}$ ) is a Hom-Jordan algebra, where " $*$ " is the product in Proposition 2.9 and $e_{1} * e_{1}=2 e_{1}, e_{2} * e_{2}=2 e_{1}, e_{3} * e_{3}=2 e_{3}, e_{1} * e_{2}=e_{2} * e_{1}=-2 e_{2}$.

Let us consider the following definitions which will be used in next sections.

Definition 2.11. Let $\left(A, \mu, \alpha_{A}\right)$ be any Hom-algebra.

(i) A Hom-module $\left(V, \alpha_{V}\right)$ is called an $A$-bimodule if it comes equipped with a left and a right structure maps on $V$ that is morphisms $\rho_{l}: A \otimes V \rightarrow V$, $a \otimes v \mapsto a \cdot v$ and $\rho_{r}: V \otimes A \rightarrow V, v \otimes a \mapsto v \cdot a$ of Hom-modules respectively.

(ii) A morphism $f:\left(V, \alpha_{V}, \rho_{l}, \rho_{r}\right) \rightarrow\left(W, \alpha_{W}, \rho_{l}^{\prime}, \rho_{r}^{\prime}\right)$ of $A$-bimodules is a morphism of the underlying Hom-modules such that

$$
f \circ \rho_{l}=\rho_{l}^{\prime} \circ\left(I d_{A} \otimes f\right) \quad \text { and } \quad f \circ \rho_{r}=\rho_{r}^{\prime} \circ\left(f \otimes I d_{A}\right) .
$$

(iii) Let $\left(V, \alpha_{V}\right)$ be an $A$-bimodule with structure maps $\rho_{l}$ and $\rho_{r}$. Then the module Hom-associator of $V$ is a trilinear map $a s_{A, V}$ defined as:

$$
\begin{aligned}
& a s_{A, V} \circ I d_{V \otimes A \otimes A}=\rho_{r} \circ\left(\rho_{r} \otimes \alpha_{A}\right)-\rho_{l} \circ\left(\alpha_{V} \otimes \mu\right), \\
& a s_{A, V} \circ I d_{A \otimes V \otimes A}=\rho_{r} \circ\left(\rho_{l} \otimes \alpha_{A}\right)-\rho_{l} \circ\left(\alpha_{A} \otimes \rho_{r}\right), \\
& a s_{A, V} \circ I d_{A \otimes A \otimes V}=\rho_{l} \circ\left(\mu \otimes \alpha_{V}\right)-\rho_{l} \circ\left(\alpha_{A} \otimes \rho_{l}\right) .
\end{aligned}
$$

Remark 2.12. The module Hom-associator given above is a generalization of the one given in [2].

Now, let consider the following notion for Hom-associative algebras.

Definition 2.13. Let $\left(A, \mu, \alpha_{A}\right)$ be a Hom-associative algebra and $\left(M, \alpha_{M}\right)$ be a Hom-module.

(i) A left Hom-associative $A$-module structure on $M$ consists of a morphism $\rho: A \otimes M \rightarrow M$ of Hom-modules, such that

$$
\rho \circ\left(\alpha_{A} \otimes \rho\right)=\rho \circ\left(\mu \otimes \alpha_{M}\right)
$$


(ii) A right Hom-associative $A$-module structure on $M$ consists of a morphism $\rho: M \otimes A \rightarrow M$ of Hom-modules, such that

$$
\rho \circ\left(\alpha_{M} \otimes \mu\right)=\rho \circ\left(\rho \otimes \alpha_{A}\right)
$$

(iii) A Hom-associative $A$-bimodule structure on $M$ consists of two structure maps $\rho_{l}: A \otimes M \rightarrow M$ and $\rho_{r}: M \otimes A \rightarrow M$ such that $\left(M, \alpha_{M}, \rho_{l}\right)$ is a left $A$-module, $\left(M, \alpha_{M}, \rho_{r}\right)$ is a right $A$-module and that the following Hom-associativity (or operator commutativity) condition holds:

$$
\rho_{l} \circ\left(\alpha_{A} \otimes \rho_{r}\right)=\rho_{r} \circ\left(\rho_{l} \otimes \alpha_{A}\right)
$$

Remark 2.14. Actually, left Hom-associative $A$-module, right Hom-associative $A$-module and Hom-associative $A$-bimodule have been already introduced in $[21,22$ where they are called left $A$-module, right $A$-module and $A$-bimodule respectively. The expressions, used in Definition 2.13 for these notions, are motivated by the unification of our terminologies.

\section{Hom-Alternative Bimodules}

In this section, we give the definition of Hom-alternative (bi)modules. We prove that from a given Hom-alternative bimodule, a sequence of this kind of bimodules can be constructed. It is also proved that a direct sum of a Homalternative algebra and a bimodule over this Hom-algebra is a Hom-alternative algebra called a split null extension of the considered Hom-algebra.

First, we start by the following notion, due to [2], where it is called a module over a left (resp. right) Hom-alternative algebra. However, we call it a Hom-alternative left (resp. right) module in this paper.

Definition 3.1. Let $\left(A, \mu, \alpha_{A}\right)$ be a Hom-alternative algebra.

(i) A left Hom-alternative $A$-module is a Hom-module $\left(V, \alpha_{V}\right)$ with a left structure map $\rho_{l}: A \otimes V \rightarrow V, a \otimes v \mapsto a \cdot v$ such that

$$
a s_{A, V}(x, y, v)=-a s_{A, V}(y, x, v) \quad \text { for all } x, y \in A \text { and } v \in V .
$$

(ii) A right Hom-alternative $A$-module is a $\operatorname{Hom}$-module $\left(V, \alpha_{V}\right)$ with a right structure map $\rho_{r}: V \otimes A \rightarrow V, v \otimes a \mapsto v \cdot a$ such that

$$
a s_{A, V}(v, x, y)=-a s_{A, V}(v, y, x) \quad \text { for all } x, y \in A \text { and } v \in V .
$$


Now, as a generalization of alternative bimodules [8, 17], one has:

Definition 3.2. Let $\left(A, \mu, \alpha_{A}\right)$ be a Hom-alternative algebra. A Homalternative $A$-bimodule is a Hom-module $\left(V, \alpha_{V}\right)$ with a (left) structure map $\rho_{l}: A \otimes V \rightarrow V, a \otimes v \mapsto a \cdot v$ and a (right) structure map $\rho_{r}: V \otimes A \rightarrow V$, $v \otimes a \mapsto v \cdot a$ such that the following equalities hold:

$$
a s_{A, V}(a, v, b)=-a s_{A, V}(v, a, b)=a s_{A, V}(b, a, v)=-a s_{A, V}(a, b, v)
$$

for all $(a, b, v) \in A^{\times 2} \times V$.

Remarks 3.3. (i) The relation (7) is equivalent to

$$
a s_{A, V}(a, v, b)=-a s_{A, V}(v, a, b)=a s_{A, V}(b, a, v)=-a s_{A, V}(b, v, a)
$$

or since the field's characteristic is 0 to

$$
a s_{A, V}(a, v, b)=-a s_{A, V}(v, a, b)=a s_{V}(b, a, v) \text { and } a s_{A, V}(a, a, v)=0 .
$$

(ii) If $\alpha_{A}=I d_{A}$ and $\alpha_{V}=I d_{V}$ then $V$ is the so-called alternative bimodule for the alternative algebra $(A, \mu)[\underline{8}$, 17].

ExAmples 3.4. Here are some examples of Hom-alternative $A$-bimodules.

(i) Let $\left(A, \mu, \alpha_{A}\right)$ be a Hom-alternative algebra. Then $\left(A, \alpha_{A}\right)$ is a Homalternative $A$-bimodule where the structure maps are $\rho_{l}(a, b)=\mu(a, b)$ and $\rho_{r}(a, b)=\mu(b, a)$. More generally, if $B$ is a two-sided Hom-ideal of $\left(A, \mu, \alpha_{A}\right)$, then $\left(B, \alpha_{A}\right)$ is a Hom-alternative $A$-bimodule where the structure maps are $\rho_{l}(a, x)=\mu(a, x)$ and $\rho_{r}(x, b)=\mu(x, b)$ for all $x \in B$ and $(a, b) \in A^{\times 2}$.

(ii) If $(A, \mu)$ is an alternative algebra and $M$ is an alternative $A$-bimodule [8] in the usual sense, then $\left(M, I d_{M}\right)$ is a Hom-alternative $\mathbb{A}$-bimodule where $\mathbb{A}=\left(A, \mu, I d_{A}\right)$ is a Hom-alternative algebra.

(iii) If $f:\left(A, \mu_{A}, \alpha_{A}\right) \rightarrow\left(B, \mu_{B}, \alpha_{B}\right)$ is a surjective morphism of Homalternative algebras, then $\left(B, \alpha_{B}\right)$ becomes a Hom-alternative $A$-bimodule via $f$, i.e, the structure maps are defined as $\rho_{l}:(a, b) \mapsto \mu_{B}(f(a), b)$ and $\rho_{r}:(b, a) \mapsto \mu_{B}(b, f(a))$ for all $(a, b) \in A \times B$. Indeed one can remark that $a s_{A, B} \circ\left(I d_{A} \otimes f \otimes I d_{A}\right)=f \circ a s_{A}$.

In order to give another example of Hom-alternative bimodules, let us consider the following 
Definition 3.5. An abelian extension of Hom-alternative algebras is a short exact sequence of Hom-alternative algebras

$$
0 \rightarrow\left(V, \alpha_{V}\right) \stackrel{i}{\longrightarrow}\left(A, \mu_{A}, \alpha_{A}\right) \stackrel{\pi}{\longrightarrow}\left(B, \mu_{B}, \alpha_{B}\right) \rightarrow 0
$$

where $\left(V, \alpha_{V}\right)$ is a trivial Hom-alternative algebra, $i$ and $\pi$ are morphisms of Hom-algebras. Furthermore, if there exists a morphism $s:\left(B, \mu_{B}, \alpha_{B}\right) \rightarrow$ $\left(A, \mu_{A}, \alpha_{A}\right)$ such that $\pi \circ s=i d_{B}$ then the abelian extension is said to be split and $s$ is called a section of $\pi$.

ExAmple 3.6. Given an abelian extension as in the previous definition, the Hom-module $\left(V, \alpha_{V}\right)$ inherits a structure of a Hom-alternative $B$-bimodule and the actions of the Hom-algebra $\left(B, \mu_{B}, \alpha_{B}\right)$ on $V$ are as follows. For any $x \in B$, there exist $\tilde{x} \in A$ such that $x=\pi(\tilde{x})$. Let $x$ acts on $v \in V$ by $x \cdot v:=\mu_{A}(\tilde{x}, i(v))$ and $v \cdot x:=\mu_{A}(i(v), \tilde{x})$. These are well-defined, as another lift $\tilde{x}^{\prime}$ of $x$ is written $\tilde{x^{\prime}}=\tilde{x}+v^{\prime}$ for some $v^{\prime} \in V$ and thus $x \cdot v=\mu_{A}(\tilde{x}, i(v))=$ $\mu_{A}\left(\tilde{x^{\prime}}, i(v)\right)$ and $v \cdot x=\mu_{A}(i(v), \tilde{x})=\mu_{A}\left(i(v), \tilde{x^{\prime}}\right)$ because $V$ is trivial. The actions property follow from the Hom-alternativity identity. In case these actions of $B$ on $V$ are trivial, one speaks of a central extension.

The following result describes a sequence of Hom-alternative bimodules by twisting the structure maps of a given bimodule over this Hom-algebra.

Proposition 3.7. Let $\left(A, \mu, \alpha_{A}\right)$ be a Hom-alternative algebra and $\left(V, \alpha_{V}\right)$ be a Hom-alternative $A$-bimodule with the structure maps $\rho_{l}$ and $\rho_{r}$. Then the maps

$$
\begin{aligned}
& \rho_{l}^{(n)}=\rho_{l} \circ\left(\alpha_{A}^{n} \otimes I d_{V}\right) \\
& \rho_{r}^{(n)}=\rho_{r} \circ\left(I d_{V} \otimes \alpha_{A}^{n}\right)
\end{aligned}
$$

give the Hom-module $\left(V, \alpha_{V}\right)$ the structure of a Hom-alternative A-bimodule that we denote by $V^{(n)}$

Proof. It is clear that $\rho_{l}^{(n)}$ and $\rho_{r}^{(n)}$ are structure maps on $V^{(n)}$. Next, observe that for all $x, y \in A$ and $v \in V$,

$$
\begin{aligned}
a s_{A, V^{(n)}}(x, v, y) & =\rho_{r}^{(n)}\left(\rho_{l}^{(n)}(x, v), \alpha_{A}(y)\right)-\rho_{l}^{(n)}\left(\alpha_{A}(x), \rho_{r}^{(n)}(v, y)\right) \\
& =\rho_{r}\left(\rho_{l}\left(\alpha_{A}^{n}(x), v\right), \alpha_{A}^{n+1}(y)\right)-\rho_{l}\left(\alpha_{A}^{n+1}(x), \rho_{r}\left(v, \alpha_{A}^{n}(y)\right)\right. \\
& =a s_{A, V}\left(\alpha_{A}^{n}(x), v, \alpha_{A}^{n}(y)\right)
\end{aligned}
$$


and similarly

$$
\begin{aligned}
& a s_{A, V^{(n)}}(v, x, y)=a s_{A, V}\left(v, \alpha_{A}^{n}(x), \alpha_{A}^{n}(y)\right), \\
& a s_{A, V^{(n)}}(y, x, v)=a s_{A, V}\left(\alpha_{A}^{n}(y), \alpha_{A}^{n}(x), v\right), \\
& a s_{A, V^{(n)}}(x, y, v)=a s_{A, V}\left(\alpha_{A}^{n}(x), \alpha_{A}^{n}(y), v\right) .
\end{aligned}
$$

Therefore, equalities of (7) in $V^{(n)}$ derive from the one in $V$.

We know that alternative algebras can be deformed into Hom-alternative algebras via an endomorphism. The following result shows that alternative bimodules can be deformed into Hom-alternative bimodules via an endomorphism. This provides a large class of examples of Hom-alternative bimodules.

Theorem 3.8. Let $(A, \mu)$ be an alternative algebra, $V$ be an alternative $A$-bimodule with the structure maps $\rho_{l}$ and $\rho_{r}, \alpha_{A}$ be an endomorphism of the alternative algebra $A$ and $\alpha_{V}$ be a linear self-map of $V$ such that $\alpha_{V} \circ \rho_{l}=$ $\rho_{l} \circ\left(\alpha_{A} \otimes \alpha_{V}\right)$ and $\alpha_{V} \circ \rho_{r}=\rho_{r} \circ\left(\alpha_{V} \otimes \alpha_{A}\right)$.

Write $A_{\alpha_{A}}$ for the Hom-alternative algebra $\left(A, \mu_{\alpha_{A}}, \alpha_{A}\right)$ and $V_{\alpha_{V}}$ for the Hom-module $\left(V, \alpha_{V}\right)$. Then the maps

$$
\tilde{\rho}_{l}=\alpha_{V} \circ \rho_{l} \quad \text { and } \quad \tilde{\rho}_{r}=\alpha_{V} \circ \rho_{r}
$$

give the Hom-module $V_{\alpha_{V}}$ the structure of a Hom-alternative $A_{\alpha_{A}}$-bimodule.

Proof. Trivially, $\tilde{\rho}_{l}$ and $\tilde{\rho}_{r}$ are structure maps on $V_{\alpha_{V}}$. The proof of $(7)$ for $V_{\alpha_{V}}$ follows directly by the fact that $a s_{A, V_{\alpha_{V}}}=\alpha_{V}^{2} \circ a s_{A, V}$ and the relation (7) in $V$.

Corollary 3.9. Let $(A, \mu)$ be an alternative algebra, $V$ be an alternative $A$-bimodule with the structure maps $\rho_{l}$ and $\rho_{r}, \alpha_{A}$ an endomorphism of the alternative algebra $A$ and $\alpha_{V}$ be a linear self-map of $V$ such that $\alpha_{V} \circ \rho_{l}=$ $\rho_{l} \circ\left(\alpha_{A} \otimes \alpha_{V}\right)$ and $\alpha_{V} \circ \rho_{r}=\rho_{r} \circ\left(\alpha_{V} \otimes \alpha_{A}\right)$.

Write $A_{\alpha_{A}}$ for the Hom-alternative algebra $\left(A, \mu_{\alpha_{A}}, \alpha_{A}\right)$ and $V_{\alpha_{V}}$ for the Hom-module $\left(V, \alpha_{V}\right)$. Then the maps

$$
\tilde{\rho}_{l}^{(n)}=\rho_{l} \circ\left(\alpha_{A}^{n+1} \otimes \alpha_{V}\right) \quad \text { and } \quad \tilde{\rho}_{r}^{(n)}=\rho_{r} \circ\left(\alpha_{V} \otimes \alpha_{A}^{n+1}\right)
$$

give the Hom-module $V_{\alpha}$ the structure of a Hom-alternative $A_{\alpha_{A}}$-bimodule for each $n \in \mathbb{N}$. 
Lemma 3.10. Let $\left(A, \mu, \alpha_{A}\right)$ be a Hom-alternative algebra and $\left(V, \alpha_{V}\right)$ be a Hom-alternative A-bimodule with the structure maps $\rho_{l}$ and $\rho_{r}$. Then the following relation

$$
a s_{A, V}(v, a, a)=0
$$

holds for all $a \in A$ and $v \in V$.

Proof. Using (7), for all $(a, b) \in A^{\times 2}$ and $v \in V$ we have $-a s_{A, V}(v, a, b)=$ $a s_{A, V}(a, v, b)$ and $a s_{A, V}(v, b, a)=-a s_{A, V}(a, b, v)$. Moreover again from (7), we get $a s_{A, V}(a, v, b)=-a s_{A, V}(a, b, v)$ and then $-a s_{A, V}(v, a, b)=a s_{A, V}(v, b, a)$. It follows that $a s_{A, V}(v, a, a)=0$ since the field $\mathbb{K}$ is of characteristic 0 .

The following result shows that a direct sum of a Hom-alternative algebra and a bimodule over this Hom-algebra, is still a Hom-alternative, called the split null extension determined by the given bimodule.

Theorem 3.11. Let $\left(A, \mu, \alpha_{A}\right)$ be a Hom-alternative algebra and $\left(V, \alpha_{V}\right)$ be a Hom-alternative $A$-bimodule with the structure maps $\rho_{l}$ and $\rho_{r}$. Defining on $A \oplus V$ the bilinear map $\tilde{\mu}:(A \oplus V)^{\otimes 2} \rightarrow A \oplus V, \tilde{\mu}(a+m, b+n):=a b+a$. $n+m \cdot b$ and the linear map $\tilde{\alpha}: A \oplus V \rightarrow A \oplus V, \tilde{\alpha}(a+m):=\alpha_{A}(a)+\alpha_{V}(m)$, then $E=(A \oplus V, \tilde{\mu}, \tilde{\alpha})$ is a Hom-alternative algebra.

Proof. The multiplicativity of $\tilde{\alpha}$ with respect to $\tilde{\mu}$ follows from the one of $\alpha$ with respect to $\mu$ and the fact that $\rho_{l}$ and $\rho_{r}$ are morphisms of Hom-modules. Next

$$
\begin{aligned}
a s_{E}(a+m, a+ & m, b+n) \\
= & \tilde{\mu}(\tilde{\mu}(a+m, a+m), \tilde{\alpha}(b+n))-\tilde{\mu}(\tilde{\alpha}(a+m), \tilde{\mu}(a+m, b+n)) \\
= & \tilde{\mu}\left(a^{2}+a \cdot m+m \cdot a, \alpha_{A}(b)+\alpha_{V}(n)\right) \\
& -\tilde{\mu}\left(\alpha_{A}(a)+\alpha_{V}(m), a b+a \cdot n+m \cdot b\right) \\
= & a^{2} \alpha_{A}(b)+a^{2} \cdot \alpha_{V}(n)+(a \cdot m) \cdot \alpha_{A}(b)+(m \cdot a) \cdot \alpha_{A}(b) \\
& -\alpha_{A}(a)(a b)-\alpha_{A}(a) \cdot(a \cdot n)-\alpha_{A}(a) \cdot(m \cdot b)-\alpha_{V}(m) \cdot(a b) \\
= & \underbrace{a s_{A}(a, a, b)}_{0}+\underbrace{a s_{V}(a, a, n)}_{0}+\underbrace{a s_{A, V}(a, m, b)+a s_{A, V}(m, a, b)}_{0}
\end{aligned}
$$

(by (1), Remarks 3.3 and (7))

$$
=0 \text {. }
$$


Similarly, we compute

$$
\begin{aligned}
& a s_{E}(a+m, b+n, b+n) \\
&=\tilde{\mu}(\tilde{\mu}(a+m, b+n), \tilde{\alpha}(b+n))-\tilde{\mu}(\tilde{\alpha}(a+m), \tilde{\mu}(b+n, b+n)) \\
&=\tilde{\mu}\left(a b+a \cdot n+m \cdot b, \alpha_{A}(b)+\alpha_{V}(n)\right) \\
&-\tilde{\mu}\left(\alpha_{A}(a)+\alpha_{V}(m), b^{2}+b \cdot n+b \cdot b\right) \\
&=(a b) \alpha_{A}(b)+(a b) \cdot \alpha_{V}(m)+(a \cdot n) \cdot \alpha_{A}(b)+(m \cdot b) \cdot \alpha_{A}(b) \\
&-\alpha_{A}(a)\left(b^{2}\right)-\alpha_{A}(a) \cdot(b \cdot n)-\alpha_{A}(a) \cdot(n \cdot b)-\alpha_{V}(m) \cdot b^{2} \\
&= \underbrace{a s_{A}(a, b, b)}_{0}+\underbrace{a s_{A, V}(a, b, n)+a s_{A, V}(a, n, b)}_{0}+\underbrace{a s_{A, V}(m, b, b)}_{0}
\end{aligned}
$$

(by (2), (7) and (8))

$$
=0 \text {. }
$$

We then conclude that $(A \oplus V, \tilde{\mu}, \tilde{\alpha})$ is a Hom-alternative algebra.

Remark 3.12. Consider the split null extension $A \oplus V$ determined by the Hom-alternative bimodule $\left(V, \alpha_{V}\right)$ of the Hom-alternative algebra $\left(A, \mu, \alpha_{A}\right)$ in the previous theorem. Write elements $a+v$ of $A \oplus V$ as $(a, v)$. Then, there is an injective homomorphism of Hom-modules $i: V \rightarrow A \oplus V$ given by $i(v)=(0, v)$ and a surjective homomorphism of Hom-modules $\pi: A \oplus V \rightarrow A$ given by $\pi(a, v)=a$. Moreover $i(V)$ is a two-sided Hom-ideal of $A \oplus V$ such that $A \oplus V / i(V) \cong A$. On the other hand, there is a morphism of Homalgebras $\sigma: A \rightarrow A \oplus V$ given by $\sigma(a)=(a, 0)$ which is clearly a section of $\pi$. Hence, we obtain the abelian split exact sequence of Hom-alternative algebras and $\left(V, \alpha_{V}\right)$ is a Hom-alternative $A$-bimodule via $\pi$.

\section{Hom-Jordan BImodules}

In this section, we study Hom-Jordan bimodules. It is observed that similar results for Hom-alternative bimodules hold for Hom-Jordan bimodules. Some of them require an additional condition. Furthermore, relations between Hom-associative bimodules and Hom-Jordan bimodules are given on the one hand, and on the other hand, relations between left (resp. right) Hom-alternative modules and left(resp. right) special Hom-Jordan modules are proved. First, we have:

Definition 4.1. Let $\left(A, \mu, \alpha_{A}\right)$ be a Hom-Jordan algebra. 
(i) A right Hom-Jordan $A$-module is a Hom-module $\left(V, \alpha_{V}\right)$ with a right structure map $\rho_{r}: V \otimes A \rightarrow V, v \otimes a \mapsto v \cdot a$ such that the following conditions hold:

$$
\begin{gathered}
\alpha_{V}(v \cdot a) \cdot \alpha_{A}(b c)+\alpha_{V}(v \cdot b) \cdot \alpha_{A}(c a)+\alpha_{V}(v \cdot c) \cdot \alpha_{A}(a b) \\
=\left(\alpha_{V}(v) \cdot b c\right) \cdot \alpha_{A}^{2}(a)+\left(\alpha_{V}(v) \cdot c a\right) \cdot \alpha_{A}^{2}(b) \\
\quad+\left(\alpha_{V}(v) \cdot a b\right) \cdot \alpha_{A}^{2}(c) \\
\alpha_{V}(v \cdot a) \cdot \alpha_{A}(b c)+\alpha_{V}(v \cdot b) \cdot \alpha_{A}(c a)+\alpha_{V}(v \cdot c) \cdot \alpha_{A}(a b) \\
=\left((v \cdot a) \cdot \alpha_{A}(b)\right) \cdot \alpha_{A}^{2}(c)+\left((v \cdot c) \cdot \alpha_{A}(b)\right) \cdot \alpha_{A}^{2}(a) \\
+\alpha_{V}^{2}(v) \cdot\left((a c) \alpha_{A}(b)\right)
\end{gathered}
$$

for all $a, b, c \in A$ and $v \in V$.

(ii) A left Hom-Jordan $A$-module is a Hom-module $\left(V, \alpha_{V}\right)$ with a left structure map $\rho_{l}: A \otimes V \rightarrow V, a \otimes v \mapsto a \cdot v$ such that the following conditions hold:

$$
\begin{aligned}
\alpha_{A}(b c) \cdot \alpha_{V}(a \cdot v)+\alpha_{A}(c a) \cdot \alpha_{V}(b \cdot v)+\alpha_{A}(a b) \cdot \alpha_{V}(c \cdot v) \\
=\alpha_{A}^{2}(a) \cdot\left(b c \cdot \alpha_{V}(v)\right)+\alpha_{A}^{2}(b) \cdot\left(c a \cdot \alpha_{V}(v)\right) \\
\quad+\alpha_{A}^{2}(c) \cdot\left(a b \cdot \alpha_{V}(v)\right) \\
\alpha_{A}(b c) \cdot \alpha_{V}(a \cdot v)+\alpha_{A}(c a) \cdot \alpha_{V}(b \cdot v)+\alpha_{A}(a b) \cdot \alpha_{V}(c \cdot v) \\
=\alpha_{A}^{2}(c) \cdot\left(\alpha_{A}(b) \cdot(a \cdot v)\right)+\alpha_{A}^{2}(a) \cdot\left(\alpha_{A}(b) \cdot(c \cdot v)\right) \\
\quad+\left((a c) \alpha_{A}(b)\right) \cdot \alpha_{V}^{2}(v)
\end{aligned}
$$

for all $a, b, c \in A$ and $v \in V$.

The following result allows to introduce the notion of right special HomJordan modules.

Theorem 4.2. Let $\left(A, \mu, \alpha_{A}\right)$ be a Hom-Jordan algebra, $\left(V, \alpha_{V}\right)$ be a Hom-module and $\rho_{r}: V \otimes A \rightarrow V, a \otimes v \mapsto v \cdot a$, be a bilinear map satisfying

$$
\alpha_{V} \circ \rho_{r}=\rho_{r} \circ\left(\alpha_{V} \otimes \alpha_{A}\right)
$$

and

$$
\alpha_{V}(v) \cdot(a b)=(v \cdot a) \cdot \alpha_{A}(b)+(v \cdot b) \cdot \alpha_{A}(a)
$$

for all $(a, b) \in A^{\times 2}$ and $v \in V$. Then $\left(V, \alpha, \rho_{r}\right)$ is a right Hom-Jordan $A$ module called a right special Hom-Jordan A-module. 
Proof. It suffices to prove $(9)$ and $(10)$. For all $(a, b) \in A^{\times 2}$ and $v \in V$, we have:

$$
\begin{aligned}
\alpha_{V}(v \cdot a) & \cdot \alpha_{A}(b c)+\alpha_{V}(v \cdot b) \cdot \alpha_{A}(c a)+\alpha_{V}(v \cdot c) \cdot \alpha_{A}(a b) \\
=\alpha_{V}(v \cdot a) \cdot \alpha_{A}(b) \alpha_{A}(c)+\alpha_{V}(v \cdot b) \cdot \alpha_{A}(c) \alpha_{A}(a) & \\
& +\alpha_{V}(v \cdot c) \cdot \alpha_{A}(a) \alpha_{A}(b) \quad(\text { multiplicativity }) \\
=( & \left.(v \cdot a) \cdot \alpha_{A}(b)\right) \cdot \alpha^{2}(c)+\left((v \cdot a) \cdot \alpha_{A}(c)\right) \cdot \alpha^{2}(b) \\
& +\left((v \cdot b) \cdot \alpha_{A}(c)\right) \cdot \alpha^{2}(a)+\left((v \cdot b) \cdot \alpha_{A}(a)\right) \cdot \alpha^{2}(c) \\
& +\left((v \cdot c) \cdot \alpha_{A}(a)\right) \cdot \alpha^{2}(b)+\left((v \cdot c) \cdot \alpha_{A}(b)\right) \cdot \alpha^{2}(a) \quad(\text { by }[14)) \\
=[ & \left.\alpha_{V}(v) \cdot a b-(v \cdot b) \alpha_{A}(a)\right] \cdot \alpha^{2}(c)+\left((v \cdot a) \cdot \alpha_{A}(c)\right) \cdot \alpha^{2}(b) \\
& +\left[\alpha_{V}(v) \cdot b c-(v \cdot c) \alpha_{A}(b)\right] \cdot \alpha^{2}(a)+\left((v \cdot b) \cdot \alpha_{A}(a)\right) \cdot \alpha^{2}(c) \\
& +\left[\alpha_{V}(v) \cdot c a-(v \cdot a) \cdot \alpha_{A}(c)\right] \cdot \alpha^{2}(b) \\
& +\left((v \cdot c) \cdot \alpha_{A}(b)\right) \cdot \alpha^{2}(a) \quad(\text { again by }[14)) \\
=( & \left.\alpha_{V}(v) \cdot b c\right) \cdot \alpha_{A}^{2}(a)+\left(\alpha_{V}(v) \cdot c a\right) \cdot \alpha_{A}^{2}(b)+\left(\alpha_{V}(v) \cdot a b\right) \cdot \alpha_{A}^{2}(c)
\end{aligned}
$$

and thus, we get (9). Finally, 10 is proved as follows:

$$
\begin{aligned}
\alpha_{V}(v \cdot a) & \cdot \alpha_{A}(b c)+\alpha_{V}(v \cdot b) \cdot \alpha_{A}(c a)+\alpha_{V}(v \cdot c) \cdot \alpha_{A}(a b) \\
= & \alpha_{V}(v \cdot a) \cdot \alpha_{A}(b) \alpha_{A}(c)+\alpha_{V}(v \cdot b) \cdot \alpha_{A}(c) \alpha_{A}(a) \\
& +\alpha_{V}(v \cdot c) \cdot \alpha_{A}(a) \alpha_{A}(b) \quad(\text { multiplicativity) } \\
= & \left((v \cdot a) \cdot \alpha_{A}(b)\right) \cdot \alpha_{A}^{2}(c)+\left((v \cdot a) \cdot \alpha_{A}(c)\right) \cdot \alpha_{A}^{2}(b) \\
& +\left((v \cdot b) \cdot \alpha_{A}(c)\right) \cdot \alpha_{A}^{2}(a)+\left((v \cdot b) \cdot \alpha_{A}(a)\right) \cdot \alpha_{A}^{2}(c) \\
& +\left((v \cdot c) \cdot \alpha_{A}(a)\right) \cdot \alpha_{A}^{2}(b)+\left((v \cdot c) \cdot \alpha_{A}(b)\right) \cdot \alpha_{A}^{2}(a) \quad(\text { by }([14)) \\
= & \left((v \cdot a) \cdot \alpha_{A}(b)\right) \cdot \alpha_{A}^{2}(c)+\left[\alpha_{V}(v) \cdot a c-\left((v \cdot c) \cdot \alpha_{A}(a)\right] \cdot \alpha_{A}^{2}(b)\right. \\
& +\left((v \cdot b) \cdot \alpha_{A}(c)\right) \cdot \alpha_{A}^{2}(a)+\left((v \cdot b) \cdot \alpha_{A}(a)\right) \cdot \alpha_{A}^{2}(c) \\
& \left.+\left((v \cdot c) \cdot \alpha_{A}(a)\right) \cdot \alpha_{A}^{2}(b)+\left((v \cdot c) \cdot \alpha_{A}(b)\right) \cdot \alpha_{A}^{2}(a) \quad \text { (again by 14) }\right) \\
= & \left((v \cdot a) \cdot \alpha_{A}(b)\right) \cdot \alpha_{A}^{2}(c)+\alpha_{V}^{2}(v) \cdot\left((a c) \alpha_{A}(b)\right) \\
& \quad-\left(\alpha_{V}(v) \cdot \alpha_{A}(b)\right) \cdot \alpha_{A}(a c)+\left((v \cdot b) \cdot \alpha_{A}(c)\right) \cdot \alpha_{A}^{2}(a) \\
& +\left((v \cdot b) \cdot \alpha_{A}(a)\right) \cdot \alpha_{A}^{2}(c)+\left((v \cdot c) \cdot \alpha_{A}(b)\right) \cdot \alpha_{A}^{2}(a) \quad \text { (again by (14)) }
\end{aligned}
$$




$$
\begin{aligned}
= & \left((v \cdot a) \cdot \alpha_{A}(b)\right) \cdot \alpha_{A}^{2}(c)+\alpha_{V}^{2}(v) \cdot\left((a c) \alpha_{A}(b)\right)-\left(\alpha_{V}(v \cdot b) \cdot \alpha_{A}(a c)\right. \\
& +\left((v \cdot b) \cdot \alpha_{A}(c)\right) \cdot \alpha_{A}^{2}(a)+\left((v \cdot b) \cdot \alpha_{A}(a)\right) \cdot \alpha_{A}^{2}(c) \\
& \left.+\left((v \cdot c) \cdot \alpha_{A}(b)\right) \cdot \alpha_{A}^{2}(a) \quad(\text { by } 13)\right) \\
= & \left((v \cdot a) \cdot \alpha_{A}(b)\right) \cdot \alpha_{A}^{2}(c)+\alpha_{V}^{2}(v) \cdot\left((a c) \alpha_{A}(b)\right)-\left((v \cdot b) \cdot \alpha_{A}(a)\right) \cdot \alpha_{A}^{2}(c) \\
& -\left((v \cdot b) \cdot \alpha_{A}(c)\right) \cdot \alpha_{A}^{2}(a)+\left((v \cdot b) \cdot \alpha_{A}(c)\right) \cdot \alpha_{A}^{2}(a) \\
& +\left((v \cdot b) \cdot \alpha_{A}(a)\right) \cdot \alpha_{A}^{2}(c)+\left((v \cdot c) \cdot \alpha_{A}(b)\right) \cdot \alpha_{A}^{2}(a) \quad(\text { by (14) }) \\
= & \left((v \cdot a) \cdot \alpha_{A}(b)\right) \cdot \alpha_{A}^{2}(c)+\left((v \cdot c) \cdot \alpha_{A}(b)\right) \cdot \alpha_{A}^{2}(a)+\alpha_{V}^{2}(v) \cdot\left((a c) \alpha_{A}(b)\right)
\end{aligned}
$$

which is 10 .

Similarly, the following result can be proved.

Theorem 4.3. Let $\left(A, \mu, \alpha_{A}\right)$ be a Hom-Jordan algebra, $\left(V, \alpha_{V}\right)$ be a Hom-module and $\rho_{l}: A \otimes V \rightarrow V, v \otimes a \mapsto a \cdot v$, be a bilinear map satisfying

$$
\alpha_{V} \circ \rho_{l}=\rho_{l} \circ\left(\alpha_{A} \otimes \alpha_{V}\right)
$$

and

$$
(a b) \cdot \alpha_{V}(v)=\alpha_{A}(a) \cdot(b \cdot v)+\alpha_{A}(b) \cdot(a \cdot v)
$$

for all $(a, b) \in A^{\times 2}$ and $v \in V$. Then $\left(V, \alpha, \rho_{l}\right)$ is a left Hom-Jordan A-module called a left special Hom-Jordan A-module.

It is well known that the plus algebra of any Hom-alternative algebra is a Hom-Jordan algebra. The next result shows that any left (resp. right) Hom-alternative module a is also a left (resp. right) module over its plus Hom-algebra.

Proposition 4.4. Let $\left(A, \mu, \alpha_{A}\right)$ be a Hom-alternative algebra and $\left(V, \alpha_{V}\right)$ be a Hom-module.

(i) If $\left(V, \alpha_{V}\right)$ is a right Hom-alternative A-module with the structure map $\rho_{r}$ then $\left(V, \alpha_{V}\right)$ is a right special Hom-Jordan $A^{+}$-module with the same structure map $\rho_{r}$.

(ii) If $\left(V, \alpha_{V}\right)$ is a left Hom-alternative A-module with the structure map $\rho_{l}$ then $\left(V, \alpha_{V}\right)$ is a left special Hom-Jordan $A^{+}$-module with the same structure map $\rho_{l}$. 
Proof. It suffices to prove (14) and (15).

(i) If $\left(V, \alpha_{V}\right)$ is a right Hom-alternative $A$-module with the structure map $\rho_{r}$, then for all $(x, y, v) \in A \times A \times V, a s_{A, V}(v, x, y)=-a s_{A, V}(v, y, x)$ by (8), i.e., $\alpha_{V}(v) \cdot(x y)+\alpha_{V}(v) \cdot(y x)=(v \cdot x) \cdot \alpha_{A}(y)+(v \cdot y) \cdot \alpha_{A}(x)$. Thus $\alpha_{V}(v) \cdot(x * y)=\alpha_{V}(v) \cdot(x y)+\alpha_{V}(v) \cdot(y x)=(v \cdot x) \cdot \alpha_{A}(y)+(v \cdot$ $y) \cdot \alpha_{A}(x)$. Therefore $\left(V, \alpha_{V}\right)$ is a right special Hom-Jordan $A^{+}$-module by Theorem 4.2 .

(ii) If $\left(V, \alpha_{V}\right)$ is a left Hom-alternative $A$-module with the structure map $\rho_{l}$, then for all $(x, y, v) \in A \times A \times V$, as $s_{A, V}(x, y, v)=-a s_{A, V}(y, x, v)$ by Remarks 3.3 and then $(x y) \cdot \alpha_{V}(v)+(y x) \cdot \alpha_{V}(v)=\alpha_{A}(x) \cdot(y \cdot v)+\alpha_{A}(y)$. $(x \cdot v)$. Thus $(x * y) \cdot \alpha_{V}(v)=(x y) \cdot \alpha_{V}(v)+(y x) \cdot \alpha_{V}(v)=\alpha_{A}(x) \cdot(y \cdot v)+$ $\alpha_{A}(y) \cdot(x \cdot v)$. Therefore $\left(V, \alpha_{V}\right)$ is a left special Hom-Jordan $A^{+}$-module by Theorem 4.3 .

Now, we give the definition of a Hom-Jordan bimodule.

Definition 4.5. Let $\left(A, \mu, \alpha_{A}\right)$ be a Hom-Jordan algebra.

A Hom-Jordan $A$-bimodule is a Hom-module $\left(V, \alpha_{V}\right)$ with a left structure map $\rho_{l}: A \otimes V \rightarrow V, a \otimes v \mapsto a \cdot v$ and a right structure map $\rho_{r}: V \otimes A \rightarrow V$, $v \otimes a \mapsto v \cdot a$, such that the following conditions hold:

$$
\begin{gathered}
\rho_{r} \circ \tau_{1}=\rho_{l}, \\
\alpha_{V}(v \cdot a) \cdot \alpha_{A}(b c)+\alpha_{V}(v \cdot b) \cdot \alpha_{A}(c a)+\alpha_{V}(v \cdot c) \cdot \alpha_{A}(a b) \\
=\left(\alpha_{V}(v) \cdot b c\right) \cdot \alpha_{A}^{2}(a)+\left(\alpha_{V}(v) \cdot c a\right) \cdot \alpha_{A}^{2}(b) \\
+\left(\alpha_{V}(v) \cdot a b\right) \cdot \alpha_{A}^{2}(c), \\
\alpha_{V}(v \cdot a) \cdot \alpha_{A}(b c)+\alpha_{V}(v \cdot b) \cdot \alpha_{A}(c a)+\alpha_{V}(v \cdot c) \cdot \alpha_{A}(a b) \\
=\left((v \cdot a) \cdot \alpha_{A}(b)\right) \cdot \alpha_{A}^{2}(c)+\left((v \cdot c) \cdot \alpha_{A}(b)\right) \cdot \alpha_{A}^{2}(a) \\
+\left((a c) \alpha_{A}(b)\right) \cdot \alpha_{V}^{2}(v),
\end{gathered}
$$

for all $a, b, c \in A$ and $v \in V$.

In term of the module Hom-associator, using the relation (16) and the fact that the structure maps are morphisms, the relations (17) and $(18)$ are respectively

$$
\begin{aligned}
\circlearrowleft_{(a, b, c)} & a s_{A, V}\left(\alpha_{A}(a), \alpha_{V}(v), b c\right)=0, \\
a s_{A, V}\left(v \cdot a, \alpha_{A}(b), \alpha_{A}(c)\right)+ & a s_{A, V}\left(v \cdot c, \alpha_{A}(b), \alpha_{A}(a)\right) \\
+ & a s_{A, V}\left(a c, \alpha_{A}(b), \alpha_{V}(v)\right)=0 .
\end{aligned}
$$


Remarks 4.6. (i) One can note that $(17)$ and $(18)$ are the same identities as $(9)$ and (10) respectively.

(ii) Since $\rho_{r} \circ \tau_{1}=\rho_{l}$, nothing is lost in dropping one of the compositions. Thus the term Hom-Jordan module can be used for Hom-Jordan bimodule.

(iii) Since the field is of characteristic 0, the identity (19) implies

$$
a s_{A, V}\left(\alpha_{A}(a), \alpha_{V}(v), a^{2}\right)=0 .
$$

(iv) If $\alpha_{A}=I d_{A}$ and $\alpha_{V}=I d_{V}$ then $V$ is reduced to the so-called Jordan module of the Jordan algebra $(A, \mu)[7,8]$.

EXAMPLES 4.7. Here are some examples of Hom-Jordan bimodules.

(i) Let $\left(A, \mu, \alpha_{A}\right)$ be a Hom-Jordan algebra. Then $\left(A, \alpha_{A}\right)$ is a HomJordan $A$-bimodule where the structure maps are $\rho_{l}=\rho_{r}=\mu$. More generally, if $B$ is a Hom-ideal of $\left(A, \mu, \alpha_{A}\right)$, then $\left(B, \alpha_{A}\right)$ is a Hom-Jordan $A$-bimodule where the structure maps are $\rho_{l}(a, x)=\mu(a, x)=\mu(x, a)=\rho_{r}(x, a)$ for all $(a, x) \in A \times B$.

(ii) If $(A, \mu)$ is a Jordan algebra and $M$ is a Jordan $A$-bimodule [8] in the usual sense then $\left(M, I d_{M}\right)$ is a Hom-Jordan $\mathbb{A}$-bimodule where $\mathbb{A}=\left(A, \mu, I d_{A}\right)$ is a Hom-Jordan algebra.

(iii) If $f:\left(A, \mu_{A}, \alpha_{A}\right) \rightarrow\left(B, \mu_{B}, \alpha_{B}\right)$ is a surjective morphism of HomJordan algebras, then $\left(B, \alpha_{B}\right)$ becomes a Hom-Jordan $A$-bimodule via $f$, i.e, the structure maps are defined by $\rho_{l}:(a, b) \mapsto \mu_{B}(b, f(a))$ and $\rho_{r}:(b, a) \mapsto$ $\mu_{B}(f(a), b)$ for all $(a, b) \in A \times B$.

As in the case of Hom-alternative algebras, in order to give another example of Hom-Jordan bimodules, let us consider the following

Definition 4.8. An abelian extension of Hom-Jordan algebras is a short exact sequence of Hom-Jordan algebras

$$
0 \rightarrow\left(V, \alpha_{V}\right) \stackrel{i}{\longrightarrow}\left(A, \mu_{A}, \alpha_{A}\right) \stackrel{\pi}{\longrightarrow}\left(B, \mu_{B}, \alpha_{B}\right) \rightarrow 0
$$

where $\left(V, \alpha_{V}\right)$ is a trivial Hom-Jordan algebra, $i$ and $\pi$ are morphisms of Hom-algebras. Furthermore, if there exists a morphism $s:\left(B, \mu_{B}, \alpha_{B}\right) \rightarrow$ $\left(A, \mu_{A}, \alpha_{A}\right)$ such that $\pi \circ s=i d_{B}$ then the abelian extension is said to be split and $s$ is called a section of $\pi$.

EXAmPLE 4.9. Given an abelian extension as in the previous definition, the Hom-module $\left(V, \alpha_{V}\right)$ inherits a structure of a Hom-Jordan $B$-bimodule 
and the actions of the Hom-algebra $\left(B, \mu_{B}, \alpha_{B}\right)$ on $V$ are as follows. For any $x \in B$, there exist $\tilde{x} \in A$ such that $x=\pi(\tilde{x})$. Let $x$ acts on $v \in V$ by $x \cdot v:=\mu_{A}(\tilde{x}, i(v))$ and $v \cdot x:=\mu_{A}(i(v), \tilde{x})$. These are well-defined, as another lift $\tilde{x^{\prime}}$ of $x$ is written $\tilde{x^{\prime}}=\tilde{x}+v^{\prime}$ for some $v^{\prime} \in V$ and thus $x \cdot v=\mu_{A}(\tilde{x}, i(v))=$ $\mu_{A}\left(\tilde{x^{\prime}}, i(v)\right)$ and $v \cdot x=\mu_{A}(i(v), \tilde{x})=\mu_{A}\left(i(v), \tilde{x^{\prime}}\right)$ because $V$ is trivial. The actions property follow from the Hom-Jordan identity. In case these actions of $B$ on $V$ are trivial, one speaks of a central extension.

The next result shows that a special left and right Hom-Jordan module has a Hom-Jordan bimodule structure under a specific condition.

Theorem 4.10. Let $\left(A, \mu, \alpha_{A}\right)$ be a Hom-Jordan algebra and $\left(V, \alpha_{V}\right)$ be both a left and a right special Hom-Jordan A-module with the structure maps $\rho_{1}$ and $\rho_{2}$ respectively such that the Hom-associativity (or operator commutativity) condition holds

$$
\rho_{2} \circ\left(\rho_{1} \otimes \alpha_{A}\right)=\rho_{1} \circ\left(\alpha_{A} \otimes \rho_{2}\right) .
$$

Define the bilinear maps $\rho_{l}: A \otimes V \rightarrow V$ and $\rho_{r}: V \otimes A \rightarrow V$ by

$$
\rho_{l}=\rho_{1}+\rho_{2} \circ \tau_{1} \quad \text { and } \quad \rho_{r}=\rho_{1} \circ \tau_{2}+\rho_{2} .
$$

Then $\left(V, \alpha_{V}, \rho_{l}, \rho_{r}\right)$ is a Hom-Jordan A-bimodule.

Proof. It is clear that $\rho_{l}$ and $\rho_{r}$ are structure maps and (16) holds. To prove relations (17) and (18), let put $\rho_{l}(a \otimes v):=a \diamond v$, i.e., $a \diamond v=a \cdot v+v \cdot a$ for all $(a, v) \in A \times V$. We have then $\rho_{r}(v \otimes a):=v \diamond a=a \cdot v+v \cdot a$ for all $(a, v) \in A \times V$. Therefore for all $(a, b, v) \in A \times A \times V$, we have

$$
\begin{aligned}
\alpha_{V}(v \diamond a) \diamond \alpha_{A}(b c)+\alpha_{V}(v \diamond b) \diamond \alpha_{A}(c a)+\alpha_{V}(v \diamond c) \diamond \alpha_{A}(a b) \\
=\alpha_{V}(v \cdot a) \cdot \alpha_{A}(b c)+\alpha_{V}(a \cdot v) \cdot \alpha_{A}(b c)+\alpha_{A}(b c) \cdot \alpha_{V}(v \cdot a) \\
\quad+\alpha_{A}(b c) \cdot \alpha_{V}(a \cdot v)+\alpha_{V}(v \cdot b) \cdot \alpha_{A}(c a)+\alpha_{V}(b \cdot v) \cdot \alpha_{A}(c a) \\
\quad+\alpha_{A}(c a) \cdot \alpha_{V}(v \cdot b)+\alpha_{A}(c a) \cdot \alpha_{V}(b \cdot v)+\alpha_{V}(v \cdot c) \cdot \alpha_{A}(a b) \\
\quad+\alpha_{V}(c \cdot v) \cdot \alpha_{A}(a b)+\alpha_{A}(a b) \cdot \alpha_{V}(v \cdot c)+\alpha_{A}(a b) \cdot \alpha_{V}(c \cdot v)
\end{aligned}
$$

(by a straightforward computation)

$$
\begin{aligned}
= & \left\{\alpha_{V}(v \cdot a) \cdot \alpha_{A}(b c)+\alpha_{V}(v \cdot b) \cdot \alpha_{A}(c a)+\alpha_{V}(v \cdot c) \cdot \alpha_{A}(a b)\right\} \\
& +\left\{\alpha_{A}(b c) \cdot\left(\alpha_{V}(v) \cdot \alpha_{A}(a)\right)+\alpha_{A}(c a) \cdot\left(\alpha_{V}(v) \cdot \alpha_{A}(b)\right)\right. \\
& \left.+\alpha_{A}(a b) \cdot\left(\alpha_{V}(v) \cdot \alpha_{A}(c)\right)\right\}+\left\{\alpha_{A}(b c) \cdot \alpha_{V}(a \cdot v)+\alpha_{A}(c a) \cdot \alpha_{V}(b \cdot v)\right.
\end{aligned}
$$




$$
\begin{aligned}
& \left.+\alpha_{A}(a b) \cdot \alpha_{V}(c \cdot v)\right\}+\left\{\left(\alpha_{A}(a) \cdot \alpha_{V}(v)\right) \cdot \alpha_{A}(b c)\right. \\
& \left.+\left(\alpha_{A}(b) \cdot \alpha_{V}(v)\right) \cdot \alpha_{A}(c a)+\left(\alpha_{A}(c) \cdot \alpha_{V}(v)\right) \cdot \alpha_{A}(a b)\right\}
\end{aligned}
$$

(rearranging terms and noting that $\rho_{1}$ and $\rho_{2}$ are morphisms)

$$
\begin{aligned}
=\{ & \left.\left(\alpha_{V}(v) \cdot b c\right) \cdot \alpha_{A}^{2}(a)+\left(\alpha_{V}(v) \cdot c a\right) \cdot \alpha_{A}^{2}(b)+\left(\alpha_{V}(v) \cdot a b\right) \cdot \alpha_{A}^{2}(c)\right\} \\
& +\left\{\left(b c \cdot \alpha_{V}(v)\right) \cdot \alpha_{A}^{2}(a)+\left(c a \cdot \alpha_{V}(v)\right) \cdot \alpha_{A}^{2}(b)+\left(a b \cdot \alpha_{V}(v)\right) \cdot \alpha_{A}^{2}(c)\right\} \\
& +\left\{\alpha_{A}^{2}(a) \cdot\left(b c \cdot \alpha_{V}(v)\right)+\alpha_{A}^{2}(b) \cdot\left(c a \cdot \alpha_{V}(v)\right)+\alpha_{A}^{2}(c) \cdot\left(a b \cdot \alpha_{V}(v)\right)\right\} \\
& +\left\{\alpha_{A}^{2}(a) \cdot\left(\alpha_{V}(v) \cdot b c\right)+\alpha_{A}^{2}(b) \cdot\left(\alpha_{V}(v) \cdot c a\right)+\alpha_{A}^{2}(c) \cdot\left(\alpha_{V}(v) \cdot a b\right)\right\}
\end{aligned}
$$

(by (9), (11) and (21))

$$
\begin{aligned}
=\{ & \left.\left(\alpha_{V}(v) \diamond b c\right) \cdot \alpha_{A}^{2}(a)+\left(\alpha_{V}(v) \diamond c a\right) \cdot \alpha_{A}^{2}(b)+\left(\alpha_{V}(v) \diamond a b\right) \cdot \alpha_{A}^{2}(c)\right\} \\
& +\left\{\alpha_{A}^{2}(a) \cdot\left(\alpha_{V}(v) \diamond b c\right)+\alpha_{A}^{2}(b) \cdot\left(\alpha_{V}(v) \diamond c a\right)+\alpha_{A}^{2}(c) \cdot\left(\alpha_{V}(v) \diamond a b\right)\right\}
\end{aligned}
$$

(by the definition of $\diamond$ )

$$
=\left(\alpha_{V}(v) \diamond b c\right) \diamond \alpha_{A}^{2}(a)+\left(\alpha_{V}(v) \diamond c a\right) \diamond \alpha_{A}^{2}(b)+\left(\alpha_{V}(v) \diamond a b\right) \diamond \alpha_{A}^{2}(c)
$$

(again by the definition of $\diamond$ ).

Therefore, we get (17). Finally, we have:

$$
\begin{aligned}
\alpha_{V}(v \diamond a) \diamond \alpha_{A}(b c)+\alpha_{V}(v \diamond b) \diamond \alpha_{A}(c a)+\alpha_{V}(v \diamond c) \diamond \alpha_{A}(a b) \\
=\alpha_{V}(v \cdot a) \cdot \alpha_{A}(b c)+\alpha_{V}(a \cdot v) \cdot \alpha_{A}(b c)+\alpha_{A}(b c) \cdot \alpha_{V}(v \cdot a) \\
+\alpha_{A}(b c) \cdot \alpha_{V}(a \cdot v)+\alpha_{V}(v \cdot b) \cdot \alpha_{A}(c a)+\alpha_{V}(b \cdot v) \cdot \alpha_{A}(c a) \\
+\alpha_{A}(c a) \cdot \alpha_{V}(v \cdot b)+\alpha_{A}(c a) \cdot \alpha_{V}(b \cdot v)+\alpha_{V}(v \cdot c) \cdot \alpha_{A}(a b) \\
+\alpha_{V}(c \cdot v) \cdot \alpha_{A}(a b)+\alpha_{A}(a b) \cdot \alpha_{V}(v \cdot c)+\alpha_{A}(a b) \cdot \alpha_{V}(c \cdot v)
\end{aligned}
$$

(by a straightforward computation)

$$
\begin{aligned}
=\{ & \left.\alpha_{V}(v \cdot a) \cdot \alpha_{A}(b c)+\alpha_{V}(v \cdot b) \cdot \alpha_{A}(c a)+\alpha_{V}(v \cdot c) \cdot \alpha_{A}(a b)\right\} \\
& +\left\{\left(\alpha_{V}(a \cdot v) \cdot \alpha_{A}(b) \alpha_{A}(c)+\left(\alpha_{V}(b \cdot v)\right) \cdot \alpha_{A}(c) \alpha_{A}(a)\right.\right. \\
& +\left(\alpha_{V}(c \cdot v) \cdot \alpha_{A}(a) \alpha_{A}(b)\right\}+\left\{\alpha_{A}(b c) \cdot \alpha_{V}(a \cdot v)+\alpha_{A}(c a) \cdot \alpha_{V}(b \cdot v)\right. \\
& \left.+\alpha_{A}(a b) \cdot \alpha_{V}(c \cdot v)\right\}+\left\{\alpha_{A}(b) \alpha_{A}(c) \cdot \alpha_{V}(v \cdot a)\right. \\
& \left.+\alpha_{A}(c) \alpha_{A}(a) \cdot \alpha_{V}(v \cdot b)+\alpha_{A}(a) \alpha_{A}(b) \cdot \alpha_{V}(v \cdot c)\right\}
\end{aligned}
$$

(rearranging terms and using the multiplicativity of $\alpha_{A}$ )

$$
=\{\underbrace{\left((v \cdot a) \cdot \alpha_{A}(b)\right) \cdot \alpha_{A}^{2}(c)}_{1}+\underbrace{\left((v \cdot c) \cdot \alpha_{A}(b)\right) \cdot \alpha_{A}^{2}(a)}_{2}+\underbrace{\alpha_{V}^{2}(v) \cdot\left((a c) \alpha_{A}(b)\right)}_{5}\}
$$




$$
\begin{aligned}
& +\{\underbrace{\left((a \cdot v) \cdot \alpha_{A}(b)\right) \cdot \alpha_{A}^{2}(c)}_{1}+\left((a \cdot v) \cdot \alpha_{A}(c)\right) \cdot \alpha_{A}^{2}(b) \\
& +\left((b \cdot v) \cdot \alpha_{A}(c)\right) \cdot \alpha_{A}^{2}(a)+\left((b \cdot v) \cdot \alpha_{A}(a)\right) \cdot \alpha_{A}^{2}(c) \\
& +\left((c \cdot v) \cdot \alpha_{A}(a)\right) \cdot \alpha_{A}^{2}(b)+\underbrace{\left((c \cdot v) \cdot \alpha_{A}(b)\right) \cdot \alpha_{A}^{2}(a)}_{2}\} \\
& +\{\underbrace{\alpha_{A}^{2}(c) \cdot\left(\alpha_{A}(b) \cdot(a \cdot v)\right)}_{3}+\underbrace{\alpha_{A}^{2}(a) \cdot\left(\alpha_{A}(b) \cdot(c \cdot v)\right)}_{4} \\
& +\underbrace{\left((a c) \alpha_{A}(b)\right) \cdot \alpha_{V}^{2}(v)}_{5}\}+\left\{\alpha_{A}^{2}(b) \cdot\left(\alpha_{A}(c) \cdot(v \cdot a)\right)\right. \\
& +\underbrace{\alpha_{A}^{2}(c) \cdot\left(\alpha_{A}(b) \cdot(v \cdot a)\right)}_{3}+\alpha_{A}^{2}(a) \cdot\left(\alpha_{A}(c) \cdot(v \cdot b)\right) \\
& +\alpha_{A}^{2}(c) \cdot\left(\alpha_{A}(a) \cdot(v \cdot b)\right)+\underbrace{\alpha_{A}^{2}(a) \cdot\left(\alpha_{A}(b) \cdot(v \cdot c)\right)}_{4} \\
& \left.+\alpha_{A}^{2}(b) \cdot\left(\alpha_{A}(a) \cdot(v \cdot c)\right)\right\} \quad(\text { by 10, 12, 14) and 15) } \\
& =\left((v \diamond a) \cdot \alpha_{A}(b)\right) \cdot \alpha_{A}^{2}(c)+\left((v \diamond c) \cdot \alpha_{A}(b)\right) \cdot \alpha_{A}^{2}(a) \\
& +\alpha_{A}^{2}(c) \cdot\left(\alpha_{A}(b) \cdot(v \diamond a)\right)+\alpha_{A}^{2}(a) \cdot\left(\alpha_{A}(b) \cdot(v \diamond c)\right) \\
& +\alpha_{V}^{2}(v) \diamond\left((a c) \alpha_{A}(b)\right)+\left((a \cdot v) \cdot \alpha_{A}(c)\right) \cdot \alpha_{A}^{2}(b) \\
& +\left((b \cdot v) \cdot \alpha_{A}(c)\right) \cdot \alpha_{A}^{2}(a)+\left((b \cdot v) \cdot \alpha_{A}(a)\right) \cdot \alpha_{A}^{2}(c) \\
& +\left((c \cdot v) \cdot \alpha_{A}(a)\right) \cdot \alpha_{A}^{2}(b)+\alpha_{A}^{2}(b) \cdot\left(\alpha_{A}(c) \cdot(v \cdot a)\right) \\
& +\alpha_{A}^{2}(a) \cdot\left(\alpha_{A}(c) \cdot(v \cdot b)\right)+\alpha_{A}^{2}(c) \cdot\left(\alpha_{A}(a) \cdot(v \cdot b)\right) \\
& +\alpha_{A}^{2}(b) \cdot\left(\alpha_{A}(a) \cdot(v \cdot c)\right) \\
& =\left((v \diamond a) \cdot \alpha_{A}(b)\right) \cdot \alpha_{A}^{2}(c)+\left((v \diamond c) \cdot \alpha_{A}(b)\right) \cdot \alpha_{A}^{2}(a) \\
& +\alpha_{A}^{2}(c) \cdot\left(\alpha_{A}(b) \cdot(v \diamond a)\right)+\alpha_{A}^{2}(a) \cdot\left(\alpha_{A}(b) \cdot(v \diamond c)\right) \\
& +\alpha_{V}^{2}(v) \diamond\left((a c) \alpha_{A}(b)\right)+\left(\alpha_{A}(a) \cdot(v \cdot c)\right) \cdot \alpha_{A}^{2}(b) \\
& +\left(\alpha_{A}(b) \cdot(v \cdot c)\right) \cdot \alpha_{A}^{2}(a)+\left(\alpha_{A}(b) \cdot(v \cdot a)\right) \cdot \alpha_{A}^{2}(c) \\
& +\left(\alpha_{A}(c) \cdot(v \cdot a)\right) \cdot \alpha_{A}^{2}(b)+\alpha_{A}^{2}(b) \cdot\left((c \cdot v) \cdot \alpha_{A}(a)\right) \\
& +\alpha_{A}^{2}(a) \cdot\left((c \cdot v) \cdot \alpha_{A}(b)\right)+\alpha_{A}^{2}(c) \cdot\left((a \cdot v) \cdot \alpha_{A}(b)\right) \\
& +\alpha_{A}^{2}(b) \cdot\left((a \cdot v) \cdot \alpha_{A}(c)\right) \quad(\text { by 21) })
\end{aligned}
$$




$$
\begin{aligned}
& =\left((v \diamond a) \cdot \alpha_{A}(b)\right) \cdot \alpha_{A}^{2}(c)+\left((v \diamond c) \cdot \alpha_{A}(b)\right) \cdot \alpha_{A}^{2}(a) \\
& +\alpha_{A}^{2}(c) \cdot\left(\alpha_{A}(b) \cdot(v \diamond a)\right)+\alpha_{A}^{2}(a) \cdot\left(\alpha_{A}(b) \cdot(v \diamond c)\right) \\
& +\alpha_{V}^{2}(v) \diamond\left((a c) \alpha_{A}(b)\right)+\underbrace{\alpha_{A}^{2}(a) \cdot\left((v \cdot c) \cdot \alpha_{A}(b)\right)}_{6} \\
& +\underbrace{\left(\alpha_{A}(b) \cdot(v \cdot c)\right) \cdot \alpha_{A}^{2}(a)}_{7}+\underbrace{\left(\alpha_{A}(b) \cdot(v \cdot a)\right) \cdot \alpha_{A}^{2}(c)}_{8} \\
& +\underbrace{\alpha_{A}^{2}(c) \cdot\left((v \cdot a) \cdot \alpha_{A}(b)\right)}_{9}+\underbrace{\left(\alpha_{A}(b) \cdot(c \cdot v)\right) \cdot \alpha_{A}^{2}(a)}_{7} \\
& +\underbrace{\alpha_{A}^{2}(a) \cdot\left((c \cdot v) \cdot \alpha_{A}(b)\right)}_{6}+\underbrace{\alpha_{A}^{2}(c) \cdot\left((a \cdot v) \cdot \alpha_{A}(b)\right)}_{9} \\
& +\underbrace{\left.\left(\alpha_{A}(b) \cdot(a \cdot v)\right) \cdot \alpha_{A}^{2}(c)\right)}_{8} \quad \text { (again by } 21) \text { ) } \\
& =\underbrace{\left((v \diamond a) \cdot \alpha_{A}(b)\right) \cdot \alpha_{A}^{2}(c)}_{10}+\underbrace{\left((v \diamond c) \cdot \alpha_{A}(b)\right) \cdot \alpha_{A}^{2}(a)}_{11} \\
& +\underbrace{\alpha_{A}^{2}(c) \cdot\left(\alpha_{A}(b) \cdot(v \diamond a)\right)}_{13}+\underbrace{\alpha_{A}^{2}(a) \cdot\left(\alpha_{A}(b) \cdot(v \diamond c)\right)}_{12} \\
& +\alpha_{V}^{2}(v) \diamond\left((a c) \alpha_{A}(b)\right)+\underbrace{\alpha_{A}^{2}(a) \cdot\left((v \diamond c) \cdot \alpha_{A}(b)\right)}_{12} \\
& +\underbrace{\left(\alpha_{A}(b) \cdot(v \diamond c)\right) \cdot \alpha_{A}^{2}(a)}_{11}+\underbrace{\left(\alpha_{A}(b) \cdot(v \diamond a)\right) \cdot \alpha_{A}^{2}(c)}_{10} \\
& +\underbrace{\alpha_{A}^{2}(c) \cdot\left((v \diamond a) \cdot \alpha_{A}(b)\right)}_{13} \\
& =\left((v \diamond a) \diamond \alpha_{A}(b)\right) \cdot \alpha_{A}^{2}(c)+\left((v \diamond c) \diamond \alpha_{A}(b)\right) \cdot \alpha_{A}^{2}(a) \\
& +\alpha_{A}^{2}(a) \cdot\left((v \diamond c) \diamond \alpha_{A}(b)\right)+\alpha_{A}^{2}(c) \cdot\left((v \diamond a) \diamond \alpha_{A}(b)\right) \\
& +\alpha_{V}^{2}(v) \diamond\left((a c) \alpha_{A}(b)\right) \\
& =\left((v \diamond a) \diamond \alpha_{A}(b)\right) \diamond \alpha_{A}^{2}(c)+\left((v \diamond c) \diamond \alpha_{A}(b)\right) \diamond \alpha_{A}^{2}(a) \\
& +\alpha_{V}^{2}(v) \diamond\left((a c) \alpha_{A}(b)\right)
\end{aligned}
$$

which is (18).

The following result will be used below. It gives a relation between Homassociative modules and special Hom-Jordan modules. 
Lemma 4.11. Let $\left(A, \mu, \alpha_{A}\right)$ be a Hom-associative algebra and $\left(V, \alpha_{V}\right)$ be a Hom-module.

(i) If $\left(V, \alpha_{V}\right)$ is a right Hom-associative $A$-module with the structure maps $\rho_{r}$ then $\left(V, \alpha_{V}\right)$ is a right special Hom-Jordan $A^{+}$-module with the same structure map $\rho_{r}$.

(ii) If $\left(V, \alpha_{V}\right)$ is a left Hom-associative $A$-module with the structure maps $\rho_{l}$ then $\left(V, \alpha_{V}\right)$ is a left special Hom-Jordan $A^{+}$-module with the same structure map $\rho_{l}$.

Proof. It also suffices to prove (14) and (15).

(i) If $\left(V, \alpha_{V}\right)$ is a right Hom-associative $A$-module with the structure map $\rho_{r}$ then for all $(x, y, v) \in A \times A \times V, \alpha_{V}(v) \cdot(a * b)=\alpha_{V}(v) \cdot(a b)+\alpha_{V}(v) \cdot(b a)=$ $(v \cdot a) \cdot \alpha_{A}(b)+(v \cdot b) \cdot \alpha_{A}(a)$ where the last equality holds by (5). Then $\left(V, \alpha_{V}\right)$ is a right special Hom-Jordan $A^{+}$-module.

(ii) If $\left(V, \alpha_{V}\right)$ is a left Hom-associative $A$-module with the structure map $\rho_{l}$ then for all $(x, y, v) \in A \times A \times V,(a * b) \cdot \alpha_{V}(v)=(a b) \cdot \alpha_{V}(v)+(b a) \cdot \alpha_{V}(v)=$ $\alpha_{A}(a) \cdot(b \cdot v)+\alpha_{A}(b) \cdot(a \cdot v)$ where the last equality holds by (4). Then $\left(V, \alpha_{V}\right)$ is a left special Hom-Jordan $A^{+}$-module.

Now, we prove that a Hom-associative module gives rise to a Hom-Jordan module for its plus Hom-algebra.

Proposition 4.12. Let $\left(A, \mu, \alpha_{A}\right)$ be a Hom-associative algebra and $\left(V, \rho_{1}, \rho_{2}, \alpha_{V}\right)$ be a Hom-associative A-bimodule. Then $\left(V, \rho_{l}, \rho_{r}, \alpha_{V}\right)$ is a Hom-Jordan $A^{+}$-bimodule where $\rho_{l}$ and $\rho_{r}$ are defined as in $(22)$.

Proof. The proof follows from Lemma 4.11, the Hom-associativity condition (6) and Theorem 4.10.

The following elementary result will be used below. It gives a property of a module Hom-associator.

Lemma 4.13. Let $\left(A, \mu, \alpha_{A}\right)$ be a Hom-Jordan algebra and $\left(V, \alpha_{V}\right)$ be an Hom-Jordan A-bimodule with the structure maps $\rho_{l}$ and $\rho_{r}$. Then

$$
\alpha_{V}^{n} \circ a s_{A, V} \circ I d_{A \otimes V \otimes A}=a s_{A, V} \circ\left(\alpha_{A}^{\otimes n} \otimes \alpha_{V}^{\otimes n} \otimes \alpha_{A}^{\otimes n}\right) .
$$


Proof. Using twice the fact that $\rho_{l}$ and $\rho_{r}$ are morphisms of Hom-modules, we get

$$
\begin{aligned}
& \alpha_{V}^{n} \circ a_{A, V} \circ I d_{A \otimes V} \otimes A \\
&=\alpha_{V}^{n} \circ\left(\rho_{r} \circ\left(\rho_{l} \otimes \alpha_{A}\right)-\rho_{l} \circ\left(\alpha_{A} \otimes \rho_{r}\right)\right) \\
&\left.=\alpha_{V}^{n} \circ \rho_{r} \circ\left(\rho_{l} \otimes \alpha_{A}\right)-\alpha_{V}^{n} \circ \rho_{l} \circ\left(\alpha_{A} \otimes \rho_{r}\right) \quad \text { (linearity of } \alpha_{V}^{n}\right) \\
&=\rho_{r} \circ\left(\alpha_{V}^{n} \circ \rho_{l} \otimes \alpha_{A}^{n+1}\right)-\rho_{l} \circ\left(\alpha_{A}^{n+1} \otimes \alpha_{V}^{n} \circ \rho_{r}\right) \\
&=\rho_{r} \circ\left(\rho_{l} \circ\left(\alpha_{A}^{n} \otimes \alpha_{V}^{n}\right) \otimes \alpha_{A}^{n+1}\right)-\rho_{l} \circ\left(\alpha_{A}^{n+1} \otimes \rho_{r} \circ\left(\alpha_{V}^{n} \otimes \alpha_{A}^{n}\right)\right) \\
&=\left(\rho_{r} \circ\left(\rho_{l} \otimes \alpha_{A}\right)-\rho_{l} \circ\left(\alpha_{A} \otimes \rho_{r}\right)\right) \circ\left(\alpha_{A}^{\otimes n} \otimes \alpha_{V}^{\otimes n} \otimes \alpha_{A}^{\otimes n}\right) \\
&=a s_{A, V} \circ\left(\alpha_{A}^{\otimes n} \otimes \alpha_{V}^{\otimes n} \otimes \alpha_{A}^{\otimes n}\right) .
\end{aligned}
$$

That ends the proof.

The next result is similar to the one of Proposition 3.7, but an additional condition is needed.

Proposition 4.14. Let $\left(A, \mu, \alpha_{A}\right)$ be a Hom-Jordan algebra and $\left(V, \alpha_{V}\right)$ be a Hom-Jordan A-bimodule with the structure maps $\rho_{l}$ and $\rho_{r}$. Suppose that there exists $n \in \mathbb{N}$ such that $\alpha_{V}^{n}=I d_{V}$. Then the maps

$$
\begin{aligned}
& \rho_{l}^{(n)}=\rho_{l} \circ\left(\alpha_{A}^{n} \otimes I d_{V}\right), \\
& \rho_{r}^{(n)}=\rho_{r} \circ\left(I d_{V} \otimes \alpha_{A}^{n}\right)
\end{aligned}
$$

give the Hom-module $\left(V, \alpha_{V}\right)$ the structure of a Hom-Jordan A-bimodule that we denote by $V^{(n)}$.

Proof. Since the structure map $\rho_{l}$ is a morphism of Hom-modules, we get:

$$
\begin{aligned}
\alpha_{V} \circ \rho_{l}^{(n)} & =\alpha_{V} \circ \rho_{l} \circ\left(\alpha_{A}^{n} \otimes I d_{V}\right) \quad(\text { by [24) }) \\
& =\rho_{l} \circ\left(\alpha_{A}^{n+1} \otimes \alpha_{V}\right) \\
& =\rho_{l} \circ\left(\alpha_{A}^{n} \otimes I d_{V}\right) \circ\left(\alpha_{A} \otimes \alpha_{V}\right) \\
& =\rho_{l}^{(n)} \circ\left(\alpha_{A} \otimes \alpha_{V}\right)
\end{aligned}
$$

Then, $\rho_{l}^{(n)}$ is a morphism. Similarly, we get that $\rho_{r}^{(n)}$ is a morphism and that (16) holds for $V^{(n)}$. Next, we compute 


$$
\begin{aligned}
\circlearrowleft_{(a, b, c)} a s_{A, V^{(n)}}\left(\alpha_{A}(a), \alpha_{V}(v), a b\right) \\
=\circlearrowleft_{(a, b, c)}\left\{\rho_{r}^{(n)}\left(\rho_{l}^{(n)}\left(\alpha_{A}(a), \alpha_{V}(v)\right), \alpha_{A}(b c)\right)-\rho_{l}^{(n)}\left(\alpha_{A}^{2}(a), \rho_{r}^{(n)}\left(\alpha_{V}(v), b c\right)\right)\right\} \\
=\circlearrowleft_{(a, b, c)}\left\{\rho_{r}\left(\rho_{l}^{(n)}\left(\alpha_{A}(a), \alpha_{V}(v)\right), \alpha_{A}^{n+1}(b c)\right)-\rho_{l}\left(\alpha_{A}^{n+2}(a), \rho_{r}^{(n)}\left(\alpha_{V}(v), b c\right)\right)\right\} \\
=\circlearrowleft_{(a, b, c)}\left\{\rho_{r}\left(\rho_{l}\left(\alpha_{A}^{n+1}(a), \alpha_{V}(v)\right), \alpha_{A}^{n+1}(b c)\right)-\rho_{l}\left(\alpha_{A}^{n+2}(a), \rho_{r}\left(\alpha_{V}(v), \alpha_{A}^{n}(b c)\right)\right\}\right. \\
=\circlearrowleft_{(a, b, c)}\left\{\rho_{r}\left(\rho_{l}\left(\alpha_{A}^{n+1}(a), \alpha_{V}(v)\right), \alpha_{A}\left(\alpha_{A}^{n}(b c)\right)\right)\right. \\
\quad \quad-\rho_{l}\left(\alpha_{A}\left(\alpha_{A}^{n+1}(a)\right), \rho_{r}\left(\alpha_{V}(v), \alpha_{A}^{n}(b c)\right)\right\} \\
=\circlearrowleft_{(a, b, c)} a s_{A, V}\left(\alpha_{A}^{n+1}(a), \alpha_{V}(v), \alpha_{A}^{n}(b c)\right) \\
\left.=\circlearrowleft_{(a, b, c)} a s_{A, V}\left(\alpha_{A}^{n+1}(a), \alpha_{V}^{n+1}(v), \alpha_{A}^{n}(b c)\right) \quad \text { (by the hypothesis } \alpha_{V}=\alpha_{V}^{n+1}\right) \\
\left.=\alpha_{V}^{n}\left(\circlearrowleft_{(a, b, c)} a s_{A, V}\left(\alpha_{A}(a), \alpha_{V}(v), b c\right)\right) \quad \text { by and the linearity of } \alpha_{V}^{n}\right) \\
=0 \quad(\text { by } 19 \text { in } V) .
\end{aligned}
$$

Then we get 19 for $V^{(n)}$. Finally remarking that

$$
\begin{aligned}
a s_{A, V^{(n)}}\left(\rho_{r}^{n}(v, a), \alpha_{A}(b), \alpha_{A}(c)\right) \\
=a s_{A, V^{(n)}}\left(v \cdot \alpha_{A}^{n}(a), \alpha_{A}(b), \alpha_{A}(c)\right) \\
=\rho_{r}^{n}\left(\rho_{r}^{n}\left(v \cdot \alpha_{A}^{n}(a), \alpha_{A}(b), \alpha_{A}^{2}(c)\right)-\rho_{r}^{n}\left(\alpha_{V}(v) \cdot \alpha_{A}^{n+1}(a), \mu\left(\alpha_{A}(b), \alpha\left({ }_{A}(c)\right)\right.\right.\right. \\
=\rho_{r}\left(\rho_{r}\left(v \cdot \alpha_{A}^{n}(a), \alpha_{A}^{n+1}(b), \alpha_{A}^{n+2}(c)\right)\right. \\
\quad \quad-\rho_{r}\left(\alpha_{V}(v) \cdot \alpha^{n+1}(a), \mu\left(\alpha_{A}^{n+1}(b), \alpha_{A}^{n+1}(c)\right)\right. \\
=\alpha_{A, V}\left(v \cdot \alpha_{A}^{n}(a), \alpha_{A}^{n+1}(b), \alpha_{A}^{n+1}(c)\right),
\end{aligned}
$$

and similarly

$$
\begin{array}{r}
a s_{A, V^{(n)}}\left(\rho_{r}^{n}(v, c), \alpha_{A}(b), \alpha_{A}(a)\right)=a s_{A, V}\left(v \cdot \alpha_{A}^{n}(c), \alpha_{A}^{n+1}(b), \alpha_{A}^{n+1}(a)\right), \\
a s_{A, V^{(n)}}\left(a c, \alpha_{A}(b), \alpha_{V}(v)\right)=a s_{A, V}\left(\alpha_{A}^{n}(a) \alpha_{A}^{n}(c), \alpha_{A}^{n+1}(b), \alpha_{V}(v)\right)
\end{array}
$$

20 is proved for $V^{(n)}$ as it follows:

$$
\begin{aligned}
& a s_{A, V^{(n)}}\left(\rho_{r}^{n}(v, a), \alpha_{A}(b), \alpha_{A}(c)\right)+a s_{A, V^{(n)}}\left(\rho_{r}^{n}(v, c), \alpha_{A}(b), \alpha_{A}(a)\right) \\
& +a s_{A, V^{(n)}}\left(a c, \alpha_{A}(b), \alpha_{V}(v)\right) \\
& =\alpha_{V}\left(v \cdot \alpha_{A}^{n}(a), \alpha_{A}^{n+1}(b), \alpha_{A}^{n+1}(c)\right)+a s_{A, V}\left(v \cdot \alpha_{A}^{n}(c), \alpha_{A}^{n+1}(b), \alpha_{A}^{n+1}(a)\right) \\
& +a s_{A, V}\left(\alpha_{A}^{n}(a) \alpha_{A}^{n}(c), \alpha_{A}^{n+1}(b), \alpha_{V}(v)\right)
\end{aligned}
$$




$$
\begin{aligned}
= & \alpha_{V}\left(v \cdot \alpha_{A}^{n}(a), \alpha_{A}\left(\alpha_{A}^{n}(b)\right), \alpha_{A}\left(\alpha_{A}^{n}(c)\right)\right) \\
& +a s_{A, V}\left(v \cdot \alpha_{A}^{n}(c), \alpha_{A}\left(\alpha_{A}^{n}(b)\right), \alpha_{A}\left(\alpha_{A}^{n}(a)\right)\right) \\
& +a s_{A, V}\left(\alpha_{A}^{n}(a) \alpha_{A}^{n}(c), \alpha_{A}\left(\alpha_{A}^{n}(b)\right), \alpha_{V}(v)\right) \\
= & 0(\text { by } 20 \text { in } V) .
\end{aligned}
$$

We conclude that $V^{(n)}$ is a Hom-Jordan $A$-bimodule.

Example 4.15. Consider the Hom-Jordan algebra $\mathcal{A}^{+}$of the Examples 2.10 and the subspace $V=\operatorname{span}\left(e_{1}, e_{3}\right)$ of $A$. Then $\left(V, \mu_{V}, \alpha_{V}\right)$ is a Homideal of $\mathcal{A}^{+}$where $\mu_{V}=\mu_{\left.A\right|_{V}}$ and $\alpha_{V}=\alpha_{\left.A\right|_{V}}$. It follows that $\left(V, \rho_{l}, \rho_{r}, \alpha_{V}\right)$ is a Hom-Jordan $\mathcal{A}^{+}$-bimodule where $\rho_{l}$ and $\rho_{r}$ are defined as in Examples 4.7. We have $\alpha_{V}^{2}=I d_{V}$, then by Proposition 4.14, the structure maps $\rho_{l}^{(2)}=$ $\rho_{l} \circ\left(\alpha_{A}^{2} \otimes I d_{V}\right)$ and $\rho_{r}^{(2)}=\rho_{r} \circ\left(I d_{V} \otimes \alpha_{A}^{2}\right)$ give the Hom-module $\left(V, \alpha_{V}\right)$ the structure of a Hom-Jordan $\mathcal{A}^{+}$-bimodule that we denote by $V^{(2)}$.

Corollary 4.16. Let $\left(A, \mu, \alpha_{A}\right)$ be a Hom-Jordan algebra and $\left(V, \alpha_{V}\right)$ be a Hom-Jordan A-bimodule with the structure maps $\rho_{l}$ and $\rho_{r}$ such that $\alpha_{V}$ is an involution. Then $\left(V, \alpha_{V}\right)$ is a Hom-Jordan A-bimodule with the structure maps $\rho_{l}^{(2)}=\rho_{l} \circ\left(\alpha_{A}^{2} \otimes I d_{V}\right)$ and $\rho_{r}^{(2)}=\rho_{r} \circ\left(I d_{V} \otimes \alpha_{A}^{2}\right)$.

Example 4.17. Consider the Hom-Jordan algebra $\mathcal{B}^{+}$of the Examples 2.10 and the subspace $V=\operatorname{span}\left(e_{1}, e_{2}\right)$ of $B$. Then $\left(V, \mu_{V}, \alpha_{V}\right)$ is a Hom-ideal of $\mathcal{B}^{+}$where $\mu_{V}=\mu_{\left.B\right|_{V}}$ and $\alpha_{V}=\alpha_{\left.B\right|_{V}}$. Therefore $\left(V, \rho_{l}, \rho_{r}, \alpha_{V}\right)$ is a HomJordan $\mathcal{B}^{+}$-bimodule where $\rho_{l}$ and $\rho_{r}$ are defined as in Examples 4.7. Note that $\alpha_{V}$ is involutive, i.e., $\alpha_{V}^{2}=I d_{V}$, then by Corollary 4.16, the structure maps $\rho_{l}^{(2)}=\rho_{l} \circ\left(\alpha_{B}^{2} \otimes I d_{V}\right)$ and $\rho_{r}^{(2)}=\rho_{r} \circ\left(I d_{V} \otimes \alpha_{B}^{2}\right)$ give the Hom-module $\left(V, \alpha_{V}\right)$ the structure of a Hom-Jordan $\mathcal{B}^{+}$-bimodule.

The following result is similar to theorem 3.8. It says that Jordan bimodules can be deformed into Hom-Jordan bimodules via an endomorphism.

Theorem 4.18. Let $(A, \mu)$ be a Jordan algebra, $V$ be a Jordan $A$-bimodule with the structure maps $\rho_{l}$ and $\rho_{r}, \alpha_{A}$ be an endomorphism of the Jordan algebra $A$ and $\alpha_{V}$ be a linear self-map of $V$ such that $\alpha_{V} \circ \rho_{l}=\rho_{l} \circ\left(\alpha_{A} \otimes \alpha_{V}\right)$ and $\alpha_{V} \circ \rho_{r}=\rho_{r} \circ\left(\alpha_{V} \otimes \alpha_{A}\right)$. Write $A_{\alpha_{A}}$ for the Hom-Jordan algebra $\left(A, \mu_{\alpha_{A}}, \alpha_{A}\right)$ and $V_{\alpha_{V}}$ for the Hom-module $\left(V, \alpha_{V}\right)$. Then the maps:

$$
\tilde{\rho}_{l}=\alpha_{V} \circ \rho_{l} \quad \text { and } \quad \tilde{\rho}_{r}=\alpha_{V} \circ \rho_{r}
$$

give the Hom-module $V_{\alpha_{V}}$ the structure of a Hom-Jordan $A_{\alpha_{A}}$-bimodule. 
Proof. It is easy to prove that the relation (16) for $V_{\alpha_{V}}$ holds and both maps $\tilde{\rho}_{l}, \tilde{\rho}_{r}$ are morphisms. Remarking that

$$
a s_{A, V_{\alpha_{V}}}=\alpha_{V}^{2} \circ a s_{A, V}
$$

we first compute

$$
\begin{aligned}
\circlearrowleft_{(a, b, c)} & a s_{A, V_{\alpha_{V}}}\left(\alpha_{A}(a), \alpha_{V}(v), \mu_{\alpha_{A}}(b, c)\right) \\
& \left.=\circlearrowleft_{(a, b, c)} \alpha_{V}^{2}\left(a s_{A, V}\left(\alpha_{A}(a), \alpha_{V}(v), \alpha_{A}(b c)\right)\right) \quad \text { (by 26) }\right) \\
& =\circlearrowleft_{(a, b, c)} \alpha_{V}^{3}\left(\left(a s_{A, V}(a, v, b c)\right) \quad(\text { by 23) })\right. \\
& =\alpha_{V}^{3}\left(\circlearrowleft_{(a, b, c)}\left(a s_{A, V}(a, v, b c)\right)\right. \\
& =0 \quad(\text { by } 19 p \text { in } V)
\end{aligned}
$$

and then, we get (19) for $V_{\alpha_{V}}$. Finally, we get

$$
\begin{aligned}
a s_{A, V_{\alpha_{V}}}\left(\tilde{\rho}_{r}(v, a), \alpha_{A}(b), \alpha_{A}(c)\right)+a s_{A, V_{\alpha_{V}}}\left(\tilde{\rho_{r}}(v, c), \alpha_{A}(b), \alpha_{A}(a)\right) & +a s_{A, V_{\alpha_{V}}}\left(\mu_{\alpha_{A}}(a, c), \alpha_{A}(b), \alpha_{V}(v)\right) \\
= & \alpha_{V}^{2}\left(a s_{A, V}\left(\tilde{\rho_{r}}(v, a), \alpha_{A}(b), \alpha_{A}(c)\right)\right)+\alpha_{V}^{2}\left(a s_{A, V}\left(\tilde{\rho_{r}}(v, c), \alpha_{A}(b), \alpha_{A}(a)\right)\right) \\
& \left.+\alpha_{V}^{2}\left(a s_{A, V}\left(\mu_{\alpha_{A}}(a, c), \alpha_{A}(b), \alpha_{V}(v)\right)\right) \quad(\text { by } 26)\right) \\
= & \alpha_{V}^{2}\left(a s_{A, V}\left(\alpha_{V}(v \cdot a), \alpha_{A}(b), \alpha_{A}(c)\right)\right) \\
& +\alpha_{V}^{2}\left(a s_{A, V}\left(\alpha_{V}(v \cdot c), \alpha_{A}(b), \alpha_{A}(a)\right)\right) \\
& +\alpha_{V}^{2}\left(a s_{A, V}\left(\alpha_{A}(a c), \alpha_{A}(b), \alpha_{V}(v)\right)\right) \\
= & \alpha_{V}^{3}\left(a s_{A, V}(v \cdot a, b, c)\right)+\alpha_{V}^{3}\left(a s_{A, V}(v \cdot c, b, a)\right) \\
& +\alpha_{V}^{3}\left(a s_{A, V}(a c, b, v)\right) \quad\left(b_{2} 23\right. \\
= & \alpha_{V}^{3}\left(a s_{A, V}(v \cdot a, b, c)+a s_{A, V}(v \cdot c, b, a)+a s_{A, V}(a c, b, v)\right) \\
= & 0 \quad\left(\operatorname{by}_{20} \text { in } V\right)
\end{aligned}
$$

which is 20) for $V_{\alpha_{V}}$. Therefore the Hom-module $V_{\alpha_{V}}$ has a Hom-Jordan $A_{\alpha_{A}}$-bimodule structure.

Corollary 4.19. Let $(A, \mu)$ be a Jordan algebra, $V$ be a Jordan $A$ bimodule with the structure maps $\rho_{l}$ and $\rho_{r}, \alpha_{A}$ be an endomorphism of the Jordan algebra $A$ and $\alpha_{V}$ be a linear self-map of $V$ such that $\alpha_{V} \circ \rho_{l}=$ $\rho_{l} \circ\left(\alpha_{A} \otimes \alpha_{V}\right)$ and $\alpha_{V} \circ \rho_{r}=\rho_{r} \circ\left(\alpha_{V} \otimes \alpha_{A}\right)$.

Moreover, suppose that there exists $n \in \mathbb{N}$ such that $\alpha_{V}^{n}=I d_{V}$. Write $A_{\alpha_{A}}$ for the Hom-Jordan algebra $\left(A, \mu_{\alpha_{A}}, \alpha_{A}\right)$ and $V_{\alpha_{V}}$ for the Hom-module 
$\left(V, \alpha_{V}\right)$. Then the maps:

$$
\tilde{\rho}_{l}^{(n)}=\rho_{l} \circ\left(\alpha_{A}^{n+1} \otimes \alpha_{V}\right) \text { and } \tilde{\rho}_{r}^{(n)}=\rho_{r} \circ\left(\alpha_{V} \otimes \alpha_{A}^{n+1}\right)
$$

give the Hom-module $V_{\alpha}$ the structure of a Hom-Jordan $A_{\alpha_{A}}$-bimodule for each $n \in \mathbb{N}$.

Proof. The proof follows from Proposition 4.14 and Theorem 4.18,

Similarly to Hom-alternative algebras, the split null extension, determined by the given bimodule over a Hom-Jordan algebra, is constructed as follows:

Theorem 4.20. Let $\left(A, \mu, \alpha_{A}\right)$ be a Hom-Jordan algebra and $\left(V, \alpha_{V}\right)$ be a Hom-Jordan A-bimodule with the structure maps $\rho_{l}$ and $\rho_{r}$. Then $(A \oplus V, \tilde{\mu}, \tilde{\alpha})$ is a Hom-Jordan algebra where

$\tilde{\mu}:(A \oplus V)^{\otimes 2} \rightarrow A \oplus V, \tilde{\mu}(a+m, b+n):=a b+a \cdot n+m \cdot b$ and $\tilde{\alpha}: A \oplus V \rightarrow A \oplus V$, $\tilde{\alpha}(a+m):=\alpha_{A}(a)+\alpha_{V}(m)$

Proof. First, the commutativity of $\tilde{\mu}$ follows from the one of $\mu$. Next, the multiplicativity of $\tilde{\alpha}$ with respect to $\tilde{\mu}$ follows from the one of $\alpha$ with respect to $\mu$ and the fact that $\rho_{l}$ and $\rho_{r}$ are morphisms of Hom-modules. Finally, we prove the Hom-Jordan identity (3) for $E=A \oplus V$ as it follows

$$
\begin{aligned}
a s_{E}(\tilde{\mu} & (x+m, x+m), \tilde{\alpha}(y+n), \tilde{\alpha}(x+m)) \\
= & \tilde{\mu}\left(\tilde{\mu}(\tilde{\mu}(x+m, x+m), \tilde{\alpha}(y+n)), \tilde{\alpha}^{2}(x+m)\right)-\tilde{\mu}(\tilde{\alpha}(\tilde{\mu}(x+m, x+m)), \\
& \tilde{\mu}(\tilde{\alpha}(y+n), \tilde{\alpha}(x+m))) \\
= & \tilde{\mu}\left(\tilde{\mu}\left(x^{2}+x \cdot m+m \cdot x, \alpha_{A}(y)-\tilde{\mu}\left(\alpha_{A}\left(x^{2}\right)+\alpha_{V}(n)\right), \alpha_{A}^{2}(x)+\alpha_{V}^{2}(m)\right)\right. \\
& \left.+\alpha_{V}(x \cdot m)+\alpha_{V}(m \cdot x), \tilde{\mu}\left(\alpha_{A}(y)+\alpha_{V}(n), \alpha_{A}(x)+\alpha_{V}(m)\right)\right) \\
= & \tilde{\mu}\left(x^{2} \alpha_{A}(y)+x^{2} \cdot \alpha_{V}(n)+(x \cdot m) \cdot \alpha_{A}(y)+(m \cdot x) \cdot \alpha_{A}(y), \alpha_{A}^{2}(x)\right. \\
& \left.+\alpha_{V}^{2}(m)\right)-\tilde{\mu}\left(\alpha_{A}^{2}\left(x^{2}\right)+\alpha_{V}(x \cdot m)+\alpha_{V}(m \cdot x), \alpha_{A}(y) \alpha_{A}(x)\right. \\
& \left.+\alpha_{A}(y) \cdot \alpha_{V}(m)+\alpha_{V}(n) \cdot \alpha_{A}(x)\right) \\
= & \left(x^{2} \alpha_{A}(y)\right) \alpha_{A}^{2}(x)+\left(x^{2} \alpha_{A}(y)\right) \cdot \alpha_{V}^{2}(m)+\left(x^{2} \cdot \alpha_{V}(n)\right) \cdot \alpha_{A}^{2}(x) \\
& \left.+\left((x \cdot m) \cdot \alpha_{A}(y)\right) \cdot \alpha_{A}^{2}(x)+\left((m \cdot x) \cdot \alpha_{A}(y)\right) \cdot \alpha_{A}^{2}(x)\right) \\
& -\alpha_{A}\left(x^{2}\right)\left(\alpha_{A}(y) \alpha_{A}(x)\right)-\alpha_{A}\left(x^{2}\right) \cdot\left(\alpha_{A}(y) \cdot \alpha_{V}(m)\right) \\
& -\alpha_{A}\left(x^{2}\right) \cdot\left(\alpha_{V}(n) \cdot \alpha_{A}(x)\right)-\alpha_{V}(x \cdot m) \cdot\left(\alpha_{A}(y) \alpha_{A}(x)\right) \\
& -\alpha_{V}(m \cdot x) \cdot\left(\alpha_{A}(y) \alpha_{A}(x)\right)
\end{aligned}
$$




$$
\begin{aligned}
= & a s_{A}\left(x^{2}, \alpha_{A}(y), \alpha_{A}(x)\right)+a s_{A, V}\left(x^{2}, \alpha_{A}(y), \alpha_{V}(m)\right) \\
& +a s_{A, V}\left(x^{2}, \alpha_{V}(n), \alpha_{A}(x)\right)+a s_{A, V}\left(x \cdot m, \alpha_{A}(y), \alpha_{A}(x)\right) \\
& +a s_{A, V}\left(m \cdot x, \alpha_{A}(y), \alpha_{A}(x)\right) \\
= & \underbrace{a s_{A, V}\left(m \cdot x, \alpha_{A}(y), \alpha_{A}(x)\right)+a s_{A, V}\left(m \cdot x, \alpha_{A}(y), \alpha_{A}(x)\right)}_{0} \\
& +\underbrace{a s_{A, V}\left(x^{2}, \alpha_{V}(n), \alpha_{A}(x)\right)+a s_{A, V}\left(x^{2}, \alpha_{A}(y), \alpha_{V}(m)\right)}_{0} \\
& +\underbrace{a s_{A}\left(x^{2}, \alpha_{A}(y), \alpha_{A}(x)\right)}_{0}=0,
\end{aligned}
$$

where the first 0 follows from (20), the second from (19) (see Remarks 4.6) and the last from the Hom-Jordan identity (3) in $A$. We conclude then that $(A \oplus V, \tilde{\mu}, \tilde{\alpha})$ is a Hom-Jordan algebra.

Similarly as Hom-alternative algebra case, let give the following:

Remark 4.21. Consider the split null extension $A \oplus V$ determined by the Hom-Jordan bimodule $\left(V, \alpha_{V}\right)$ for the Hom-Jordan algebra $\left(A, \mu, \alpha_{A}\right)$ in the previous theorem. Write elements $a+v$ of $A \oplus V$ as $(a, v)$. Then there is an injective homomorphism of Hom-modules $i: V \rightarrow A \oplus V$ given by $i(v)=(0, v)$ and a surjective homomorphism of Hom-modules $\pi: A \oplus V \rightarrow A$ given by $\pi(a, v)=a$. Moreover, $i(V)$ is a Hom-ideal of $A \oplus V$ such that $A \oplus V / i(V) \cong A$. On the other hand, there is a morphism of Hom-algebras $\sigma: A \rightarrow A \oplus V$ given by $\sigma(a)=(a, 0)$ which is clearly a section of $\pi$. Hence, we obtain the abelian split exact sequence of Hom-Jordan algebras and $\left(V, \alpha_{V}\right)$ is a Hom-Jordan bimodule for $A$ via $\pi$.

\section{REFERENCES}

[1] H. Ataguema, A. Makhlouf, S.D. Silvestrov, Generalization of $n$ ary Nambu algebras and beyond, J. Math. Phys. 50 (8) (2009), 083501, 15 pp.

[2] I. BaKayoko, B. Manga, Hom-alternative modules and Hom-Poisson comodules. arXiv: $1411.7957 \mathrm{v} 1$

[3] S. Eilenberg, Extensions of general algebras, Ann. Soc. Polon. Math. 21 (1948), $125-34$.

[4] F. Gürsey, C.-H. Tze, On The Role of Division, Jordan and Related Algebras in Particle Physics, World Scientific Publishing Co., Inc., River Edge, NJ, 1996. 
[5] N. Huang, L.Y. Chen, Y. Wang, Hom-Jordan algebras and their $\alpha^{k}$ (a,b,c)-derivations, Comm. Algebra 46 (6) (2018), 2600-2614.

[6] J.T. Hartwig, D. Larsson, S.D. Silvestrov, Deformations of Lie algebras using $\sigma$-derivations, J. Algebra 292 (2006), 314-361.

[7] N. Jacobson, General representation theory of Jordan algebras, Trans. Amer. Math. Soc. 70 (1951), 509-530.

[8] N. Jacobson, Structure of alternative and Jordan bimodules, Osaka Math. J. 6 (1954), 1-71.

[9] P. Jordan, J. von Neumann, E. Wigner, On an algebraic generalization of the quantum mechanical formalism, Ann. of Math. (2) 35 (1934), 29-64.

[10] D. Larsson, S.D. Silvestrov, Quasi-Hom-Lie algebras, central extensions and 2-cocycle-like identities, J. Algebra 288 (2005), 321-344.

[11] D. Larsson, S.D. Silvestrov, Quasi-Lie algebras, in "Noncommutative Geometry and Representation Theory in Mathematical Physics", Contemp. Math., 391, Amer. Math. Soc., Providence, RI, 2005, 241-248.

[12] D. Larsson, S.D. Silvestrov, Quasi-deformations of $\mathfrak{s l}_{2}(\mathbb{F})$ using twisted derivations, Comm. Algebra 35 (12) (2007), 4303-4318.

[13] A. Makhlouf, S. Silvestrov, Notes on 1-parameter formal deformations of Hom-associative and Hom-Lie algebras, Forum Math. 22 (4) (2010), 715739.

[14] A. Makhlouf, Hom-Alternative algebras and Hom-Jordan algebras, Int. Electron. J. Algebra 8 (2010), 177-190.

[15] A. Makhlouf, S.D. Silvestrov, Hom-algebra structures, J. Gen. Lie Theory Appl. 2 (2008), 51-64.

[16] S. OKubo, "Introduction to Octonion and other Non-associative Algebras in Physics", Cambridge University Press, Cambridge, 1995.

[17] R.D. Schafer, Representations of alternative algebras, Trans. Amer. Math. Soc. 72 (1952), 1-17.

[18] Y. Sheng, Representation of hom-Lie algebras, Algebr. Represent. Theory 15 (6) (2012), 1081-1098.

[19] T.A. Springer, F.D. Veldkamp, "Octonions, Jordan Algebras, and Exceptional Groups", Springer-Verlag, Berlin, 2000.

[20] J. Tits, R.M. Weiss, "Moufang Polygons", Springer-Verlag, Berlin, 2002.

[21] D. YAU, Hom-algebras and homology. arXiv:0712.3515v1

[22] D. YAU, Module Hom-algebras. arXiv:0812.4695v1

[23] D. YAU, Hom-Maltsev, Hom-alternative and Hom-Jordan algebras, Int. Electron. J. Algebra 11 (2012), 177-217.

[24] A. Zahary, A. Makhlouf, Structure and classification of Hom-associative algebras. arXiv:1906.04969V1 [math.RA] 
